HH

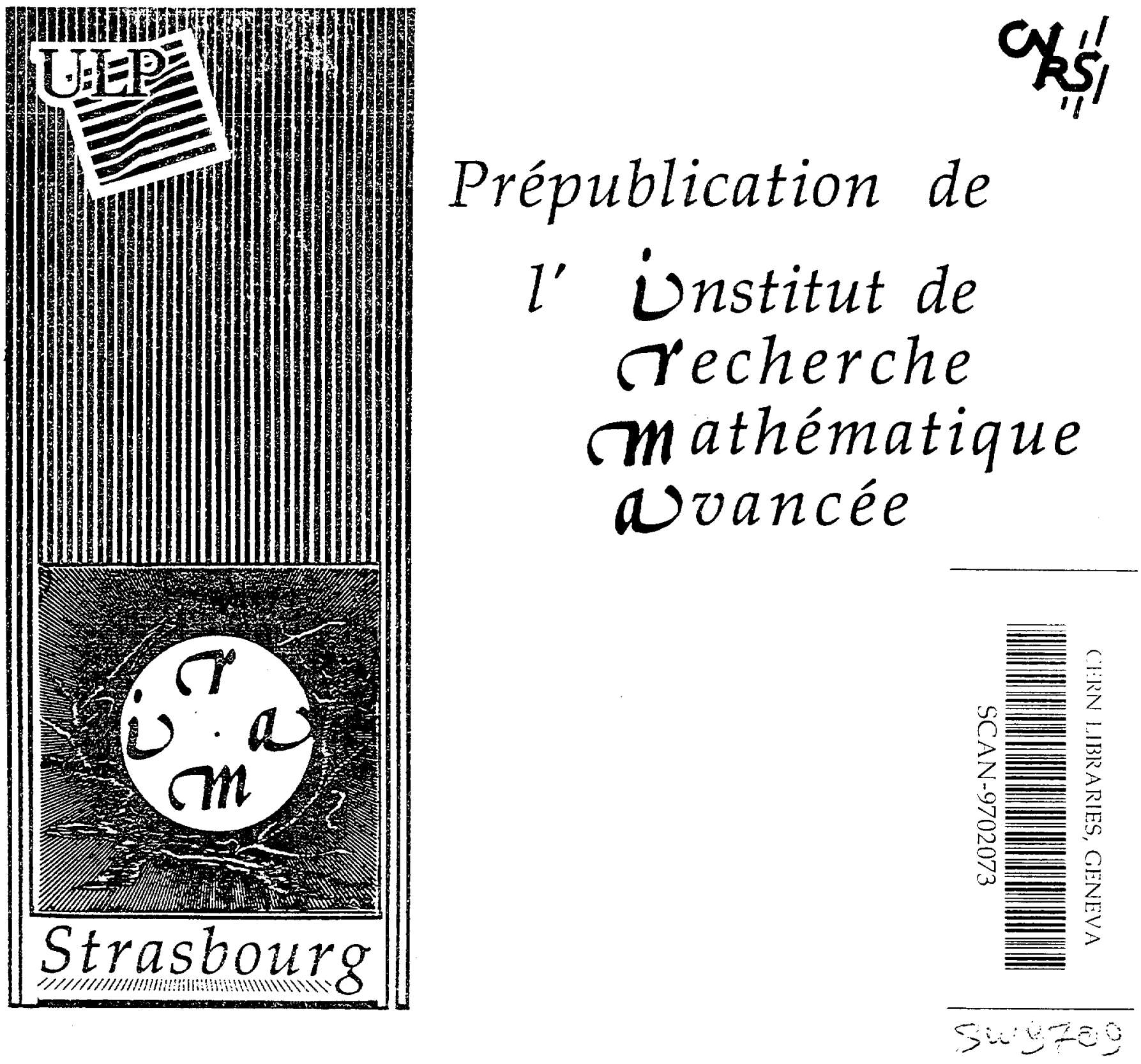

GALOIS COHOMOLOGY IN DEGREE THREE AND HOMOGENEOUS VARIETIES

Emmanuel PEYRE 

INSTITUT DE RECHERCHE MATHÉMATIQUE AVANCÉE

Université Louis Pasteur et C.N.R.S. (URA 01)

7 , rue René Descartes

67084 STRASBOURG Cedex

\section{GALOIS COHOMOLOGY IN DEGREE THREE \\ AND HOMOGENEOUS VARIETIES}

Emmanuel PEYRE

AMS Classification : 12G05, 14M15, 14C15.

Mots clés : Cohomologie galoisienne, variétés de drapeaux généralisées, groupes de Chow. 


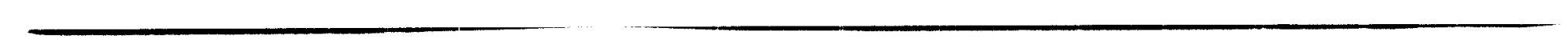




\title{
GALOIS COHOMOLOGY IN DEGREE THREE AND HOMOGENEOUS VARIETIES
}

\author{
EMMANUEL PEYRE
}

March 12, 1996

\begin{abstract}
The main result of this paper is the following generalization of a result of the author on products of Severi-Brauer varieties. Let $G$ be a semi-simple linear algebraic group over a field $k$. Let $V$ be a generalized flag variety under $G$. Then there exist finite extensions $k_{i}$ of $k$ for $1 \leqslant i \leqslant m$, elements $\alpha_{i}$ in $\mathrm{Br} k_{i}$ and a natural exact sequence

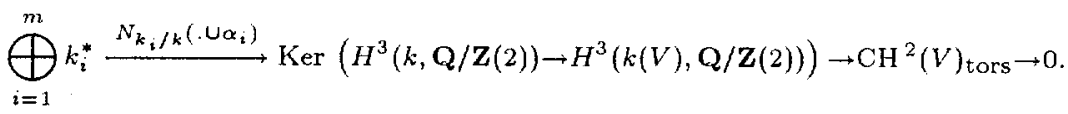

After giving a more explicit expression of the second morphism in a particular case, we apply this result to get $k$-negligible classes for a group which is a central extension of an $\mathbf{F}_{2}$ vector space by another, under the hypothesis that $k$ is field of characteristic different from 2 and contains a fourth root of unity.
\end{abstract}

\section{Contents}

1. Introduction

2. Notation and statement of the main result

3. K-cohomology

4. Explicit description of the Hochschild-Serre spectral sequence for hypercohomology

5. Proof of the main statement

6. Connection with Panin's result

7. A few examples

7.1. The low-dimensional cases

7.2. The case of orthogonal groups

8. An explicit expression in a particular case

9. Application to negligible classes

9.1. Products of generic Severi-Brauer varieties

9.2. Connection with negligible classes

9.3. An explicit example

References 


\section{INTRODUCTION}

The central result of this paper is a generalization of a previous result of the author about products of Severi-Brauer varieties [Pe2, theorem 4.1]. Let $V$ be a generalized flag variety for a linear algebraic group $G$ over a field $k$. Then the Picard group of $V$ over a separable closure of $k$ has a canonical globally invariant basis $B$. Let $E$ be the étale algebra corresponding to the Galois set $B$. There exists a class $\alpha$ in the Braner group of $E$ and an exact sequence

$$
E^{*} \stackrel{N_{E / k}(\cup \alpha)}{\longrightarrow} \operatorname{Ker}\left(H^{3}(k, \mathrm{Q} / \mathrm{Z}(2)) \rightarrow H^{3}(k(V), \mathrm{Q} / \mathrm{Z}(2))\right) \rightarrow \mathrm{CH}^{2}(V)_{\text {tors }} \rightarrow 0 .
$$

In fact the part of the kernel given by the image of $E^{*}$ comes from the kernel for Brauer groups in the following sense: this image is the union, for $k^{\prime}$ describing the separable finite extensions of $k$ of the corestriction of the group $k^{\prime *} \cup \operatorname{Ker}\left(\operatorname{Br} k^{\prime} \rightarrow \operatorname{Br} k^{\prime}(V)\right)$. This kernel has been studied independantly by Merkur'ev in [Me].

After giving a few applications of this result and describing an explicit construction of the second map in a particular case, we turn to the second topic of this paper, the problem of $k$-negligible classes in the third cohomology group of some meta-abelian groups $G$. The idea is to remplace the field of invariants $k(W)^{G}$ by a function field $K(V)$ where $K$ is purely transcendantal over $k$ and $V$ a generalized flag variety over $K$ for which we may apply the previous results of the paper. We then use this machinery to get a $k$-negligible class in $H^{3}(G, \mathbf{Q} / \mathbf{Z})$ where $G$ is a central extension of an $\mathbf{F}_{2}$ vector space by another and $k$ a field of characteristic different from 2 and containing the fourth roots of one.

Some of these results have been stated with shortened proofs in [Pe4].

\section{Notation and STATEMENT OF THE MAIN RESUlt}

Notation. For any field $L, \bar{L}$ denotes an algebraic closure of $L$ and $L^{s}$ the separable closure of $L$ in $\bar{L}$. For any discrete $\operatorname{Gal}\left(L^{s} / L\right)$-module $M$, set

$$
H^{i}(L, M)=H^{i}\left(\operatorname{Gal}\left(L^{s} / L\right), M\right) \text {. }
$$

If the characteristic of $L$ does not divide $n, \mu_{n}$ denotes the group of $n$-th roots of unity in $L^{s}$. If $p$ is the exponential characteristic of $L$, which coincides with the usual one if it is different from 0 and is 1 otherwise, $i$ a positive integer and $j$ an integer, we put (see [Kah])

$$
H^{i}\left(L,(\mathbf{Q} / \mathbf{Z})^{\prime}(j)\right)=\varliminf_{(p, n)=1}^{\lim _{\longrightarrow}} H^{i}\left(L, \mu_{n}^{\otimes j}\right)
$$

and, if $j=0,1$ or 2 ,

$$
H^{i}\left(L, \mathbf{Q}_{p} / \mathbf{Z}_{p}(j)\right)=\varliminf_{\longrightarrow} H^{i-j}\left(L, K_{j}\left(L^{s}\right) / p^{r}\right) .
$$

Then if $j=0,1$ or 2 we put

$$
H^{i}(L, \mathbf{Q} / \mathbf{Z}(j))=H^{i}\left(L,(\mathbf{Q} / \mathbf{Z})^{\prime}(j)\right) \oplus H^{i}\left(L, \mathbf{Q}_{p} / \mathbf{Z}_{p}(j)\right) .
$$

If $L^{\prime}$ is a finite separable field extension of $L$ then $N_{L^{\prime} / L}$ denote the corestriction morphism from the Galois cohomology of $L^{\prime}$ to that of $L$.

If $V$ is a variety over $L$ then $L(V)$ is the function field of $V$. If moreover $L^{\prime}$ is a field extension of $L$ then $V_{L^{\prime}}$ denotes the product $V \times{ }_{\operatorname{Spec}} L \operatorname{Spec} L^{\prime}$ and $V^{s}$ the variety $V_{L^{s}}$. For any nonnegative integer $i, V^{(i)}$ denotes the set of points of codimension $i$ in $V$. The sheaf $\mathcal{K}_{i}$ is the sheaf on $V$ for Zariski topology corresponding to the presheaf mapping $U$ to $K_{i}(U)$, the $i$-th group of Quillen's $K$-theory. If $V$ is smooth, the codimension of support defines a decreasing filtration on $K^{i}(V)$ which is denoted by $K^{i}(V)^{j}$. The quotient $K^{i}(V)^{j} / K^{i}(V)^{j+1}$ is denoted by $K^{i}(V)^{(j / j+1)}$ 
A generalized flag variety is a projective variety which is homogeneous under the action of a connected linear algebraic group $G$ and such that $V^{s}$ is isomorphic to the quotient of $G^{s}$ by a standard parabolic subgroup. Without loss of generality, we may assume that the group $G$ is semi-simple and simply connected.

From now on, $G$ will denote an semi-simple linear algebraic group over a field $k$ and $V$ a generalized flag variety under $G$. We denote by $\mathcal{G}$ the Galois group of $k^{s}$ over $k$.

Theorem 2.1. With notation as above, the Picard group of $V^{s}$ is a $\mathcal{G}$ permulation module. This means that there exist subgroups $\mathcal{H}_{i}$ of $\mathcal{G}$ of finite index such that $\mathrm{Pic} V$ 's isomorphic to $\bigoplus_{i=1}^{n} \mathrm{Z}\left[\mathcal{G} / \mathcal{H}_{i}\right]$. Let $k_{i}$ be the corresponding fields. Then for any $i$ there is a class $\alpha_{i}$ of $\mathrm{Br} k_{i}$ such that:

(i) In the natural exact sequence

$$
\operatorname{Pic} V \rightarrow \operatorname{Pic}\left(V^{s}\right)^{\mathcal{G}} \stackrel{\rho}{\rightarrow} \operatorname{Br} k \rightarrow \operatorname{Br} k(V)
$$

the natural generator of $\mathbf{Z}\left[\mathcal{G} / \mathcal{H}_{i}\right]^{\mathcal{G}}$ is sent to $N_{k_{2} / k}\left(\alpha_{i}\right)$ by $\rho$.

(ii) The homology of the complex

$$
\bigoplus_{i=1}^{m} k_{i}^{*} \stackrel{N_{k_{i} / k}\left(U \alpha_{i}\right)}{\longrightarrow} H^{3}(k, \mathbf{Q} / \mathbf{Z}(2)) \rightarrow H^{3}(k(V), \mathbf{Q} / \mathbf{Z}(2))
$$

is canonically isomorphic to the torsion subgroup of $\mathrm{CH}^{2}(V)$. In particular this homology is finite.

Remark 2.2. Merkur'ev has proved in $[\mathrm{Me}]$ that the kernel of the first map in $(\mathcal{C})$ coincides with $H^{1}\left(V, \mathcal{K}_{2}\right)$.

\section{3. $\mathcal{K}$-COHomology}

We shall now consider the $\mathcal{G}$-module structure of the $\mathcal{K}$-cohomology groups of $V^{s}$.

We fix a parabolic subgroup $P$ of $G^{s}$ such that $V^{s}$ is isomorphic to $P \backslash G^{s}$. Let $B$ be a Borel subgroup of $G^{s}$ contained in $P, T$ be a maximal torus in $B$, $\Phi$ be the root system of $T$ in $G^{s}$. The letter $\Delta$ denotes the basis of $\Phi$ corresponding to $B$. Let $I$ be the subset of $\Delta$ corresponding to $P$ (see [Bo, page 234]). For any subset $J$ of $\Delta$, the corresponding parabolic subgroup is denoted by $P_{J}$. Let $V_{J}$ be the homogeneous variety $P_{J} \backslash G^{*}$ and $\pi_{J}$ be the canonical projection $G^{s} \rightarrow V_{J}$. The subgroup generated by the $s_{\alpha}$ for $\alpha \in J$ is denoted by $W_{J}$ and the set of the unique elements of minimal length in the classes $W_{J} w$ for $w$ in $W$ by $W^{J}$. Let $w_{J}$ be the longest element in $W_{J}$.

Let $\mathfrak{g}$ be the Lie algebra of $G^{s}$ and for any $\alpha \in \Phi$ let

$$
\mathfrak{g}_{\alpha}=\left\{X \in \mathfrak{g} \mid \forall t \in T\left(k^{s}\right), \operatorname{Ad}(t)(X)=\alpha(t) X\right\} .
$$

The unique subgroup of $G^{s}$ normalized by $T$ and having $\mathfrak{g}_{\alpha}$ as Lie algebra is denoted by $U_{\alpha}$. If $w$ belongs to $W$, then $U_{w}^{\prime}$ is the subgroup of $G^{s}$ generated by the $U_{\gamma}$ where $\gamma$ describes

$$
\{\delta \in \Phi \mid \delta>0 \text { and } w \delta<0\} .
$$

By Bruhat's decomposition, $G^{s}$ is the disjoint union of the double classes $C(w)=B w B$ for $w \in W$. Moreover by [Bo, theorem 14.12] there are isomorphisms

$$
\begin{aligned}
B \times U_{w}^{\prime} & \check{\rightarrow} B w B \\
(b, u) & \mapsto b w u
\end{aligned}
$$


and by [Bo, theorem 21.29], the sets $\pi_{J}(C(w))$ give a cellular decomposition of $V_{J}$. Moreover the isomorphism (1) yields an isomorphism from $U_{u}^{\prime}$ to $\pi_{J}(C(w))$. In particular by [Bki, chapitre VI, $\mathrm{n}^{\mathrm{O}} 1.6$, page 158 , corollaire 2],

$$
\operatorname{dim}\left(\pi_{J}(C(w))\right)=\#\{\gamma \in \Phi \mid \gamma>0 \text { and } w \gamma<0\}=l(w)
$$

Let $X_{J, w}=\overline{\pi_{J}(C(w))}$. By $\left[\mathrm{Fu}\right.$, example 1.9.1], the Chow group $\mathrm{CH}_{i}\left(V_{J}\right)$ is generated by the classes $\left[X_{J, w}\right]$ for

$$
w \in W_{i}^{J}=\left\{w \in W^{J} \mid l(w)=i\right\}
$$

and by [De, corollaire page 69] these classes form a basis when $j=\emptyset$. We shall now generalize this result.

Proposition 3.1. With notation as above, $\bigoplus_{i, j \geqslant 0} H^{i}\left(V_{J}, \mathcal{K}_{i+j}\right)$ is a free $\bigoplus_{j \geqslant 0} K_{j} k^{s}$ module with a canonical basis given by the classes $\left[X_{J, w}\right]$ in $H^{i}\left(V_{J}, \mathcal{K}_{i}\right)$ for w in $W_{\text {dim } V_{J}-i}^{J}$ where $i$ describes $\left\{0, \ldots, \operatorname{dim} V_{J}\right\}$. Moreover the map $w \mapsto w_{J} w_{\Delta}$ induces a bijection $w \longmapsto \bar{u}$ from $W_{i}^{J}$ to $W_{\operatorname{dim} V_{J}-i}^{J}$ and in the Chow ring $\bigoplus_{i \geqslant 0} H^{i}\left(V_{J}, \mathcal{K}_{i}\right)$ one has for any $w \in W_{i}^{J}$ and any $w^{\prime} \in W_{\mathrm{dim} V_{y}-i}^{J}$

$$
\left[X_{J, w}\right] \cdot\left[X_{J, w^{\prime}}\right]=\delta_{\bar{w}, w^{\prime}}\left[X_{J, e}\right]
$$

where $\left[X_{J, \varepsilon}\right]$ is the class of a point.

We first state two corollaries of this proposition.

Corollary 3.2. The $\mathcal{G}$-lattices $H^{i}\left(V^{s}, \mathcal{K}_{i}\right)$ are permutation modules.

Proof. Let $C_{\mathrm{eff}}^{i} \subset H^{i}\left(V^{s}, \mathcal{K}_{i}\right) \otimes \mathbf{Q}$ be the cone of classes of effective divisors. Then $\left[X_{I_{w}}\right]$ belongs to this cone. On the other hand, let

$$
\alpha=\sum_{w \in W_{i}^{I}} n_{w}\left[X_{I, w}\right]
$$

be an element of $C_{\text {eff }}^{\operatorname{dim}} V-i$. Then, let $E$ be an effective divisor representing a multiple of $\alpha$. By [Fu, page 441] for any $w$ belonging to $W_{\operatorname{dim} V-i}^{I}$, there exists $g$ in $G\left(s^{3}\right)$ such that the intersection of $X_{I, w} \cdot g$ with $E$ is a reunion of points. Thus one has $\left[X_{I, w}\right] .[E] \geqslant 0$ and hence $\left[X_{I, w}\right] . \alpha \geqslant 0$. But, by the proposition, for any $w \in W_{i}^{I}, n_{w}=\left[X_{I, \bar{w}}\right] . \alpha$. Therefort: we get

$$
C_{\mathrm{eff}}^{\operatorname{dim} V-i}=\sum_{w \in W_{I}^{I}} \mathbf{Q}_{\geqslant 0}\left[X_{I, w}\right] .
$$

The action of $\mathcal{G}$ on $H^{i}\left(V^{s}, \mathcal{K}_{i}\right) \otimes \mathbf{Q}$ leaves $C_{\text {eff }}^{i}$ globally invariant. Its faces of dimension one remain also invariant and thus the basis $\left(\left[X_{I, w}\right]\right)_{w \in W_{d i m}^{I} v-i}$ is also globally invariant.

Corollary 3.3. For any positive integer $i$, one has

$$
H^{1}\left(\mathcal{G}, H^{i}\left(V^{s}, \mathcal{K}_{i+1}\right)\right)=0 .
$$

Proof. By proposition 3.1, there are isomorphisms

$$
H^{i}\left(V^{s}, \mathcal{K}_{i+1}\right) \stackrel{\sim}{\rightarrow} k^{*} \otimes \mathbf{z} H^{i}\left(V^{s}, \mathcal{K}_{r i}\right),
$$

but the right term is a permutation module by corollary 3.2 . Let $\mathcal{G}_{i}$ be the subgroups of $\mathcal{G}$ such that $H^{i}\left(V^{s}, \mathcal{K}_{i}\right) \stackrel{\sim}{\rightarrow} \bigoplus_{i=1}^{m} \mathbf{Z}\left[\mathcal{G} / \mathcal{G}_{i}\right]$. Then by Shapiro's lemma

$$
H^{i}\left(V^{s}, \mathcal{K}_{i+1}\right) \sim \bigoplus_{i=1}^{m} H^{1}\left(\mathcal{G}_{i}, k^{s *}\right) \text {. }
$$


But by Hilbert's theorem 90 , the groups $H^{1}\left(\mathcal{G}_{i}, k^{s *}\right)$ are trivial.

Proof of proposition 3.1. Let us first prove the assertions concerning the Chow ring. By [Fu, example 1.9.1], the classes $\left[X_{J, w}\right]$ generate the $\mathbf{Z}$-module $\bigoplus_{i \in \mathbb{N}} H^{i}\left(V_{J}, \mathcal{K}_{i}\right)$. Let $w$ belong to $W_{i}^{J}$ and let us show that $w^{\prime}=w_{J} w w_{\Delta}$ belongs to $W_{\operatorname{dim} V_{J}-i}^{J}$. By [Bki, chapitre IV, $\S 1$, exercice 3],

$$
l\left(w_{J} w\right)=l\left(w_{J}\right)+l(w)=\operatorname{dim} V_{\emptyset}-\operatorname{dim} V_{J}+l(w)
$$

and by [Bki, chapitre VI, $\mathrm{n}^{\circ} 1.6$, page 158 , corollaire 3$]$,

$$
l\left(w_{J} w w_{\Delta}\right)=l\left(w_{\Delta}\right)-l\left(w_{J} w\right)=\operatorname{dim} V_{J}-l(w)
$$

Let us write $w^{\prime}$ in the form $w_{1} w_{2}$ with $w_{1} \in W_{J}$ and $w_{2} \in W^{J}$. Then

$$
l\left(w_{J} w^{\prime}\right)=l\left(w_{J} w_{1} w_{2}\right)=l\left(w_{J}\right)-l\left(w_{1}\right)+l\left(w_{2}\right)
$$

Thus we have

$$
l(w)=l\left(w_{J} w^{\prime} w_{\Delta}\right)=l\left(w_{\Delta}\right)-l\left(w_{J}\right)+l\left(w_{1}\right)-l\left(w_{2}\right)=\operatorname{dim} V_{J}+l\left(w_{1}\right)-l\left(w_{2}\right) .
$$

But $l\left(w^{\prime}\right)=l\left(w_{1}\right)+l\left(w_{2}\right)=\operatorname{dim}\left(V_{J}\right)-l(w)$ and we get $2 l\left(w_{1}\right)=0$, which yields that $w_{1}$ is trivial.

Let us now consider elements $w$ of $W_{i}^{J}$ and $w^{\prime}$ of $W_{\operatorname{dim} V_{J}-i}^{J}$. We denote by $\pi_{\emptyset, J}$ the canonical projection from $V_{\emptyset}$ to $V_{J}$. By [Bo, proposition 21.29], one has the equality

$$
\pi_{\emptyset, J}\left(\pi_{\emptyset}\left(C\left(w_{J} w\right)\right)\right)=\pi_{J}(C(w))
$$

since $l\left(w_{J} w\right)=l\left(w_{J}\right)+l(w)=\operatorname{dim}\left(V_{\emptyset}\right)-\operatorname{dim}\left(V_{J}\right)+l(w)$, we get that $\pi_{\emptyset, J}^{-1}\left(X_{J, w}\right)$ is equal to $X_{\mathfrak{b}, w_{J} w}$. Then, using [De, $\S 3.3$, proposition 1] and [Fu, proposition 8.3], we obtain the following equalities

$$
\begin{aligned}
{\left[X_{J, w}\right]\left[X_{J, w^{\prime}}\right] } & =\left[X_{J, w}\right] \cdot \pi_{\emptyset, J_{*}}\left(\left[X_{\emptyset, w^{\prime}}\right]\right) \\
& =\pi_{\emptyset, J_{*}}\left(\pi_{\emptyset, J}^{*}\left(\left[X_{J, w}\right]\right) \cdot\left[X_{\emptyset, w^{\prime}}\right]\right) \\
& =\pi_{\emptyset, J_{*}}\left(\left[X_{\emptyset, w^{\prime}, w}\right] \cdot\left[X_{\emptyset, w^{\prime}}\right]\right) \\
& =\pi_{\emptyset, J_{*}}\left(\delta_{w_{J} w, w^{\prime} w_{\Delta}}\left[X_{\emptyset, e}\right]\right) \\
& =\delta_{\bar{w}, w^{\prime}}\left[X_{J, e}\right] .
\end{aligned}
$$

Thus the classes $\left[X_{J, w}\right]$ for $w \in W^{J}$ give a basis of the Chow ring and the intersection formula is proved.

Let $N$ be the cardinal of $W^{J}$. Let us now choose a bijection from $\{1, \ldots, N\}$ to $W^{J}$ such that $i \leqslant i^{\prime}$ implies $l\left(w_{i}\right) \geqslant l\left(w_{i}^{\prime}\right)$. Then for any $i$ between 1 and $N$ we denote by $O_{i}$ the open set $\bigcup_{j \leqslant i} \pi_{J}\left(C\left(w_{j}\right)\right)$. We shall prove by induction on $i$ that for any $i$ such that $1 \leqslant i \leqslant N$ the $\bigoplus_{j \geqslant 0} K_{j} k^{s}$-module $\bigoplus_{j, l \geqslant 0} H^{l}\left(O_{i}, \mathcal{K}_{i+j}\right)$ is free with a basis given by the classes

$$
\left[\overline{\pi_{J}\left(C\left(w_{j}\right)\right)}\right] \in H^{\operatorname{dim} V_{J}-l\left(w_{j}\right)}\left(V_{J}, \mathcal{K}_{\mathrm{dim} V_{J}-l\left(w_{j}\right)}\right)
$$

for $j$ in $\{1, \ldots, i\}$. For $i=1$, the open set $O_{1}$ is isomorphic to an affine space and the assertion is a consequence of the homotopy theorem for $\mathcal{K}$-cohomology (see [Sh, theorem 2.4]). Let us assume the result for $i-1$. Then $U_{i}=O_{i}-O_{i-1}=\pi_{J}\left(C\left(w_{i}\right)\right)$ is isomorphic to an affine space of dimension $l\left(w_{i}\right)$. By the homotopy theorem one has

$$
H^{p}\left(U_{i}, \mathcal{K}_{q}\right)= \begin{cases}K_{q} k^{s} & \text { if } p=0, \\ 0 & \text { otherwise. }\end{cases}
$$


But there are long exact sequences

$$
\ldots H^{p-d}\left(U_{i}, \mathcal{K}_{q-d}\right) \rightarrow H^{p}\left(O_{i}, \mathcal{K}_{q}\right) \rightarrow H^{p}\left(O_{i-1}, \mathcal{K}_{q}\right) \stackrel{\partial^{p, q}}{\longrightarrow} H^{p+1-d}\left(U_{i}, \mathcal{K}_{q-d}\right) \rightarrow \ldots
$$

where $d=\operatorname{dim} V_{J}-l\left(w_{i}\right)$. For $q=p$ one has in particular

$$
\ldots \stackrel{\partial_{i}^{p-1, p}}{\longrightarrow} H^{p-d}\left(U_{i}, \mathcal{K}_{p-d}\right) \rightarrow H^{p}\left(O_{i}, \mathcal{K}_{p}\right) \rightarrow H^{p}\left(O_{i-1}, \mathcal{K}_{p}\right) \rightarrow 0
$$

But, if $p>d$ the group $H^{p-d}\left(U_{i}, \mathcal{K}_{p-d}\right)$ is trivial and $\partial_{i}^{p-1, p}=0$. On the other hand, if $p=d$ then

$$
\operatorname{rk}\left(H^{p}\left(V_{J}, \mathcal{K}_{p}\right)\right)=\# W_{p}^{J}=\sum_{\left\{i \mid l\left(w_{1}\right)=\operatorname{dim} V_{J}-p\right\}} \operatorname{rk}\left(\text { Coker } \partial_{i}^{p-1, p}\right),
$$

and the maps $\partial_{i}^{p, p-1}$ are trivial. But the morphisms $\partial_{i}$ are $K_{*} k^{s}$ linear and by induction hypothesis $H^{p}\left(O_{i-1}, \mathcal{K}_{p+*}\right)$ is a free $K_{*} k^{s}$-module. Therefore all maps $\partial_{i}^{p, q}$ are trivial. Hence we obtain the following commutative diagram the lines of which are exact:

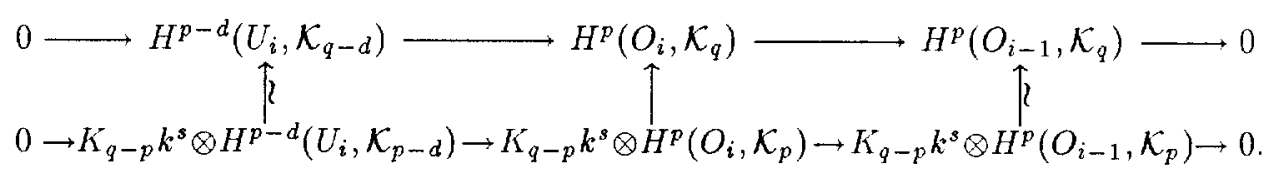

Therefore the vertical line in the middle is also an isomorphism.

Concerning the Chow groups of $V$ we simply note the following facts:

Proposition 3.4. Let $k^{\prime}$ be a separable finite extension of $k$ which splits $G$.

(i) The group $K_{0}(V)$ is without torsion. We may identify it with its image in $K_{0}\left(V_{k^{\prime}}\right)$.

(ii) One has the inclusions

$$
\left[k^{\prime}: k\right] K_{0}\left(V_{k^{\prime}}\right)^{i} \cap K_{0}(V) \subset K_{0}(V)^{i} \subset K_{0}\left(V_{k^{\prime}}\right)^{i} \cap K_{0}(V) .
$$

(iii) The kernel of the surjective map

$$
\mathrm{CH}^{i}(V) \rightarrow K_{0}(V)^{(i / i+1)}
$$

is killed by $(i-1)$ ! and $\left[k^{\prime}: k\right]$.

(iv) $K_{0}(V)^{i}=K_{0}\left(V_{k^{\prime}}\right)^{i} \cap K_{0}(V)$ if $i=1$ or 2.

(v) $\mathrm{CH}^{2}(V)_{\text {tors }} \stackrel{\sim}{\rightarrow}\left(K_{0}\left(V_{k^{\prime}}\right)^{3} \cap K_{0}(V)\right) / K_{0}(V)^{3}$.

Proof. The assertion (i) is a consequence of Panin's result [Pa, theorem 4.2]. The assertions (ii), (iii) and (iv) are proved as the similar assertions of [Pe2, proposition 3.8]. For the last assertion, since $\mathrm{CH}^{2}\left(V_{k^{\prime}}\right)$ is without torsion and

$$
\operatorname{Ker}\left(\mathrm{CH}^{2}(V) \rightarrow \mathrm{CH}^{2}\left(V_{k^{\prime}}\right)\right)
$$

is killed by $\left[k^{\prime}: k\right]$, one has

$$
\begin{aligned}
\mathrm{CH}^{2}(V)_{\text {tors }} & =\operatorname{Ker}\left(\mathrm{CH}^{2}(V) \rightarrow \mathrm{CH}^{2}\left(V_{k^{\prime}}\right)\right) \\
& =\operatorname{Ker}\left(K_{0}(V)^{2} / K_{0}(V)^{3} \rightarrow K_{0}\left(V_{k^{\prime}}\right)^{2} / K_{0}\left(V_{k^{\prime}}\right)^{3}\right) \\
& =\left(K_{0}(V)^{2} \cap K_{0}\left(V_{k^{\prime}}\right)^{3}\right) / K_{0}(V)^{3} \\
& =\left(K_{0}(V) \cap K_{0}\left(V_{k^{\prime}}\right)^{3}\right) / K_{0}(V)^{3} . \square
\end{aligned}
$$




\section{Explicit description of the Hochschild-SERre spectral SEQUENCE for HYPERCOHOMOLOGY}

The construction of the morphism from the homology of the complex $(\mathcal{C})$ to the group $\mathrm{CH}^{2}(V)_{\text {tors }}$ involves the Hochschild-Serre spectral sequence for relative hypercohomology groups of $k^{s}(V) / k^{s}$ with coefficients in the Lichtenbaum complex $\Gamma$ (2) (see [Kah, page $68])$. Therefore we shall now give an explicit construction of this spectral sequence and then check that it is compatible with corestriction. In fact, this construction is a simple generalization of the one of Hochschild and Serre and the proof of the compatibility is quite straightforward but we include them for self-completeness.

For any group $G$ and any $G$-module $M, C^{i}(G, M)$ denotes the group of normalized n-cochains; this means the group of functions $f: G^{n} \rightarrow M$ such that $f\left(g_{1}, \ldots, g_{n}\right)$ is trivial whenever one of the $g_{i}$ is $e$. Let $G$ be a group and $K$ be a normal subgroup of $G$. Let $\left(M^{\bullet}, \delta\right)$ be a bounded complex of $G$-modules. We consider the group

$$
A(G)=\bigoplus_{\substack{i \geqslant 0 \\ j \in \mathbf{z}}} C^{i}\left(G, M^{j}\right)
$$

which is a bicomplex for the derivations $d^{\prime}$ and $d^{\prime \prime}$ where

$$
d^{\prime}: C^{i}\left(G, M^{j}\right) \rightarrow C^{i+1}\left(G, M^{j}\right)
$$

is the standard non homogeneous coboundary operator and

$$
d^{\prime \prime}: C^{i}\left(G, M^{j}\right) \rightarrow C^{i}\left(G, M^{j+1}\right)
$$

is $(-1)^{i} \delta_{*}$. We put $A^{(i, j)}(G)=C^{i}\left(G, M^{j}\right)$ if $i \geqslant 0$ and $j \in \mathbf{Z}$ and $A^{(i, j)}(G)=0$ otherwise. As in [HS, page 119] one defines a decreasing filtration on $A$ as follows: $A_{l}^{(i, j)}(G)$ is $A^{(i, j)}(G)$ if $l \leqslant 0, A_{l}^{(i, j)}(G)$ is the set of $i$-cochains $\gamma: G^{i} \rightarrow M^{j}$ such that $\gamma\left(g_{1}, \ldots, g_{i}\right)$ depends only on $g_{1}, \ldots, g_{i-l}, g_{i-l+1} K, \ldots, g_{i} K$ if $0 \leqslant l \leqslant i$ and is 0 otherwise. Then we put

$$
A_{l}^{n}(G)=\bigoplus_{i+j=n} A_{l}^{(i, j)}(G) \text { and } A_{l}(G)=\bigoplus_{n \in Z} A_{l}^{n}(G)
$$

By [HS, page 119] this filtration is compatible with $d^{\prime}$. The compatibility with $d^{\prime \prime}$ is clear. Therefore it is compatible with the total complex

$$
\left(\bigoplus_{n \in \mathbf{Z}} \bigoplus_{i+j=n} A^{(i, j)}(G), d=d^{\prime}+d^{\prime \prime}\right)
$$

Moreover there is a natural morphism

$$
\phi_{l}: A_{l}^{(i, j)}(G) \rightarrow C^{l}\left(G / K, C^{i-l}\left(K, M^{j}\right)\right)
$$

obtained by restricting the first $i-l$ coordinates of an element of $A_{l}^{(i, j)}(G)$ to $K$.

We recall that the corresponding spectral sequence is then defined by

$$
Z_{r}^{p, q}(G)=\left\{a \in A_{p}^{p+q}(G) \mid d a \in A_{p+r}^{p+q+1}(G)\right\}
$$

and

$$
E_{r}^{p, q}(G / K)=Z_{r}^{p, q}(G) /\left(Z_{r-1}^{p+1, q-1}(G)+d\left(Z_{r-1}^{p-r+1, q+r-2}(G)\right)\right)
$$


Lemma 4.1. With notation as above, the map $\phi_{p}$ induces an isomorphism

$$
E_{1}^{p, q}(G / K) \stackrel{\sim}{\rightarrow} C^{p}\left(G / K, \mathbf{H}^{q}\left(K, M^{\bullet}\right)\right)
$$

and

$$
E_{2}^{p, q}(G / K) \stackrel{\sim}{\rightarrow} H^{p}\left(G / K, \mathbf{H}^{q}\left(K, M^{\bullet}\right)\right)
$$

Proof. By construction, if we denote also by $d^{\prime}$ the map induced on $C^{p}\left(G / K, C^{q}\left(K, M^{j}\right)\right)$ by the standard coboundary operator for $K$, then $\phi_{l}$ commutes with $d^{\prime \prime}$. Let $d^{\prime \prime}$ be defined as $(-1)^{p+q} \delta_{*}$ on $C^{p}\left(G / K, C^{q}\left(K, M^{j}\right)\right)$. Then $\phi_{l}$ and $d^{\prime \prime}$ commute. Thus $\phi_{l}$ induces a canonical map

$$
E_{1}^{p, q}(G / K) \rightarrow C^{p}\left(G / K, \mathbf{H}^{q}\left(K, M^{\bullet}\right)\right) .
$$

Let us check that it is an injection. Let us consider pairs $(\boldsymbol{f}, \boldsymbol{u})$ with

$$
\boldsymbol{f}=\left(f_{i}\right)_{i \geqslant 0} \in \bigoplus_{i \geqslant 0} A_{p}^{(i, p+q-i)}(G) \text { and } \boldsymbol{u}=\left(u_{i}\right)_{i \geqslant 0} \in \bigoplus_{i \geqslant 0} C^{p}\left(G / K, C^{i-p}\left(K, M^{p+q-1-i}\right)\right)
$$

such that $d \boldsymbol{f}$ belongs to $A_{p+1}^{p+q+1}(G)$ and $\phi_{p}(\boldsymbol{f})=d(\boldsymbol{u})$. It is enough to prove by an increasing induction on $m$ that for any such pair which verifies

$$
\forall i \in \mathbf{N}, i \geqslant m \Rightarrow u_{i}=0 \text { and } f_{i} \in A_{p+1}^{(i, p+q-i)}(G),
$$

there exists $\boldsymbol{h} \in A_{p}^{p+q-1}(G)$ such that

$$
\boldsymbol{f}-d \boldsymbol{h} \in A_{p+1}^{p+q}(G) .
$$

It is true if $m$ is zero. Let us assume it is true for $m-1$. Let $(\boldsymbol{f}, \boldsymbol{u})$ be a pair which verify the condition for $m$. By definition of $d$ one has that

$$
d^{\prime}\left(f_{m}\right) \in A_{p+1}^{(m+1, p+q-m)}(G) \text { and } \phi_{p}\left(f_{m}\right)=d^{\prime}\left(u_{m-1}\right) .
$$

By [HS, pages 121 and 122], there exists a cochain $h_{m-1}$ in $A_{p}^{(m-1, p+q-m)}(G)$ such that

$$
\phi_{p}\left(h_{m-1}\right)=u_{m-1} \text { and } f_{m}-d^{\prime}\left(h_{m-1}\right) \in A_{p+1}^{(m, p+q-m)}(G) \text {. }
$$

let $\boldsymbol{f}^{\prime}=\boldsymbol{f}-d\left(h_{m-1}\right)$. Then $\boldsymbol{f}^{\prime} \in Z_{1}^{p, q}(G)$ and

$$
\phi_{p}\left(\boldsymbol{f}^{\prime}\right)=\phi_{p}(\boldsymbol{f})-\phi_{p} \circ d\left(h_{m-1}\right)=d(\boldsymbol{u})-d_{\circ} \phi_{p}\left(h_{m-1}\right) .
$$

It is enough to check the result for $f^{\prime}$. But

$$
f_{m}^{\prime}=f_{m}-d^{\prime}\left(h_{m-1}\right) \in A_{(p+1)}^{(m, p+q-m)}(G)
$$

and $\phi_{p}\left(\boldsymbol{f}^{\prime}\right)=d\left(\boldsymbol{u}^{\prime}\right)$ where

$$
u_{i}^{\prime}= \begin{cases}u_{i} & \text { if } i \leqslant m-1 \\ 0 & \text { otherwise. }\end{cases}
$$

Thus we may apply the induction hypothesis.

Let us now prove the surjectivity. Let

$$
(\boldsymbol{u})=\left(u_{i}\right)_{i \geqslant 0} \in \bigoplus_{i \geqslant 0} C^{p}\left(G / K, C^{i-p}\left(K, M^{p+q-i}\right)\right)
$$

be such that $d(\boldsymbol{u})=0$. We shall prove by a decreasing induction on $i$ the existence of

$$
\left(f_{j}\right)_{j \geqslant i} \in \bigoplus_{j \geqslant i} C^{j}\left(G, M^{p+q-j}\right)
$$


such that if $j>i$

$$
d^{\prime \prime}\left(f_{j}\right)+d^{\prime}\left(f_{j-1}\right) \in A_{p+1}^{(j, p+q-j+1)}(G)
$$

and if $j \geqslant i$, we have $\phi_{p}\left(f_{j}\right)=u_{j}$. It is true for $i$ big enough since $M^{\bullet}$ is bounded. Let us assume it is true for $i+1$. Then we have $\phi_{p}\left(f_{i+1}\right)=u_{i+1}$. But $d^{\prime \prime}\left(u_{i+1}\right)+d^{\prime}\left(u_{i}\right)=0$. Thus

$$
\phi_{p}\left(d^{\prime \prime}\left(f_{i+1}\right)\right)=d^{\prime \prime}\left(\phi_{p}\left(f_{i+1}\right)\right)=d^{\prime}\left(-u_{i}\right)
$$

Moreover one has

$$
d^{\prime}\left(d^{\prime \prime}\left(f_{i+1}\right)\right)=d^{\prime \prime}\left(-d^{\prime \prime}\left(f_{i+2}\right)-d^{\prime}\left(f_{i+1}\right)\right) \in A_{p+1}^{(i+2, p+q-i)}(G)
$$

Therefore, by [HS, pages 121 and 122], there exists $f_{i} \in A_{p}^{(i, p+q-i)}(G)$ such that

$$
\phi_{p}\left(f_{i}\right)=u_{i} \text { and } d^{\prime}\left(f_{i}\right)+d^{\prime \prime}\left(f_{i+1}\right) \in A_{p+1}^{(i+1, p+q-i)}(G)
$$

and the result is proved for $i$. Thus we get an element

$$
f=\left(f_{i}\right)_{i \geqslant 0} \in \bigoplus_{i \geqslant 0} A_{p}^{(i, p+q-i)}(G)
$$

such that

$$
\phi_{p}(f)=\boldsymbol{u} \text { and } d(f) \in A_{p+1}^{p+q}(G) .
$$

This proves the first assertion of the lemma.

It remains to show that we have a commutative diagram:

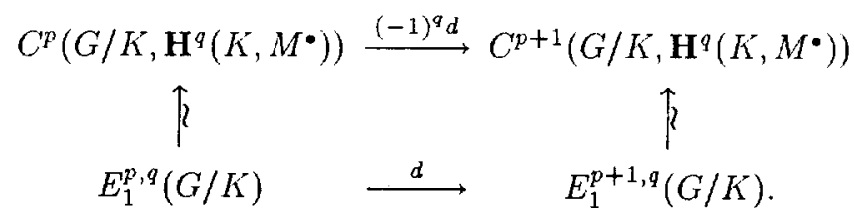

As in [HS, page 123] we consider the partial coboundary operators defined, for any $f \in C^{p+q-1}\left(G, M^{n}\right)$ by

$$
\begin{aligned}
\delta_{p-1}^{\prime} f\left(\alpha_{1}, \ldots, \alpha_{q}, \beta_{1}, \ldots, \beta_{p}\right)= & \alpha_{1} . f\left(\alpha_{2}, \ldots, \alpha_{q}, \beta_{1}, \ldots, \beta_{p}\right) \\
& +\sum_{k=1}^{q-1}(-1)^{k} f\left(\alpha_{1}, \ldots, \alpha_{k} \alpha_{k+1}, \ldots, \alpha_{q}, \beta_{1}, \ldots, \beta_{p}\right) \\
& +(-1)^{q} f\left(\alpha_{1}, \ldots, \alpha_{q-1}, \beta_{1}, \ldots, \beta_{p}\right)
\end{aligned}
$$

and

$$
\begin{aligned}
\partial_{p-1} f\left(\alpha_{1}, \ldots, \alpha_{q}, \beta_{1}, \ldots, \beta_{p}\right)= & \beta_{1} f\left(\beta_{1}^{-1} \alpha_{1} \beta_{1}, \ldots, \beta_{1}^{-1} \alpha_{q} \beta_{1}, \beta_{2}, \ldots, \beta_{p}\right) \\
& +\sum_{k=1}^{p-1}(-1)^{k} f\left(\alpha_{1}, \ldots, \alpha_{q}, \beta_{1}, \ldots, \beta_{k} \beta_{k+1}, \ldots, \beta_{p}\right) \\
& +(-1)^{p} f\left(\alpha_{1}, \ldots, \alpha_{q}, \beta_{1}, \ldots, \beta_{p-1}\right) .
\end{aligned}
$$

For any subset $S=\left\{s_{1}, \ldots, s_{p}\right\}$ of $\{1, \ldots, p+q\}$ with $s_{1}<\cdots<s_{p}$, let $S^{*}=\left\{s_{1}^{*}, \ldots, s_{q}^{*}\right\}$ be its complement with $s_{1}^{*}<\cdots<s_{q}^{*}$. Write $i^{*}=s_{i}^{*}-i$ and $\nu(S)=\sum_{i=1}^{q} i^{*}$ and set $b_{0}=1$ and $b_{k}=\beta_{1} \ldots \beta_{k}$ for $1 \leqslant k \leqslant p$. As in [HS, page 123] one defines for any $g \in C^{p+q}\left(G, M^{n}\right)$

$$
g_{s}\left(\alpha_{1}, \ldots, \alpha_{p}, \beta_{1}, \ldots, \beta_{q}\right)=g\left(\gamma_{1}, \ldots, \gamma_{p+q}\right)
$$


where $\gamma_{s_{i}}=\beta_{i}$ for $1 \leqslant i \leqslant p$ and $\gamma_{s_{i}^{*}}=b_{i^{*}}^{-1} \alpha_{i} b_{i^{*}}$ for $1 \leqslant i \leqslant q$ and $g_{\{p\}}=\sum_{S}(-1)^{\nu(S)} g_{S}$ where $S$ ranges over all the subsets of $p$ elements from $(1, \ldots, p+q)$. By [HS, page 123, proposition 2], for any $f$ in $C^{p+q-1}\left(G, M^{n}\right)$ one has

$$
\left(d^{\prime} f\right)_{(p)}=\delta_{p-1}^{\prime}\left(f_{(p)}\right)+(-1)^{q} \partial_{p-1}\left(f_{(p-1)}\right) .
$$

Let $\delta_{q}^{\prime \prime}$ be defined by

$$
\left(\delta_{q}^{\prime \prime} f\right)\left(\alpha_{1}, \ldots, \alpha_{q}, \beta_{1}, \ldots, \beta_{p}\right)=(-1)^{p+q} \delta\left(f\left(\alpha_{1}, \ldots, \alpha_{q}, \beta_{1}, \ldots, \beta_{p}\right)\right)
$$

and $\delta_{p}: A^{p+q}(G) \rightarrow A^{p+q+1}(G)$ by $\delta_{p}=\delta_{p}^{\prime}+\delta_{p}^{\prime \prime}$. Then

$$
\forall f \in A^{(p+i, q-i)}(G),(d f)_{(p+1)}=\delta_{p}\left(f_{(p+1)}\right)+(-1)^{i} \partial_{p}\left(f_{(p)}\right) .
$$

Let $D_{p}: A(G) \rightarrow A(G)$ be defined as $(-1)^{i} \partial_{p}$ on $A^{(p+i, q-i)}(G)$. Then $D_{p}$ induces on $C^{p}\left(G / K, C^{i}\left(K, M^{q-i}\right)\right)$ a map $D_{p}$ which commutes with the maps $d^{\prime}$ and $d^{\prime \prime}$ defined at the begimning of the proof and induces $(-1)^{q} d$ on $C^{p}\left(G / K, \mathbf{H}^{q}\left(K, M^{\bullet}\right)\right)$. On the other hand, for any $g$ belonging to $A_{p}^{(p+i, q-i)}(G)$, the natural image of $\phi_{p}(g)$ in $C^{p}\left(G, C^{i}\left(K, M^{q-i}\right)\right)$ coincides with the restriction of $g_{(p)}$ to $K^{i} \times G^{p}$. Thus by (3) the map induced by $d$ on

$$
\operatorname{Im}\left(C^{p}\left(G / K, \mathbf{H}^{q}\left(K, M^{\bullet}\right)\right) \rightarrow C^{p}\left(G, \mathbf{H}^{q}\left(K, M^{\bullet}\right)\right)\right)
$$

coincides with the one defined by $D_{p}$ and this implies the commutativity of the diagram (2).

Lemma 4.2. Let $K \subset H \subset G$ be three groups such that $K$ is normal in $G$ and $H$ is of finite index in $G$ and let $M^{\bullet}$ be a bounded complex of $G$-modules. Then the HochschildSerre spectral sequences

$$
E_{2}^{p, q}(H / K)=H^{p}\left(H / K, \mathbf{H}^{q}\left(K, M^{\bullet}\right)\right) \Rightarrow \mathbf{H}^{p+q}\left(H, M^{\bullet}\right)
$$

and

$$
E_{2}^{p, q}(G / K)=H^{p}\left(G / K, \mathbf{H}^{q}\left(K, M^{\bullet}\right)\right) \Rightarrow \mathbf{H}^{p+q}\left(G, M^{\bullet}\right)
$$

are compatible with the corestriction map. More precisely, there are natural maps

$$
\text { Cores }_{i}: E_{i}^{p, q}(H / K) \rightarrow E_{i}^{p, q}(G / K)
$$

such that one has commutative diagrams

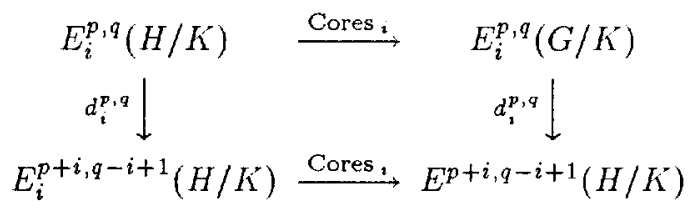

and

$$
\begin{array}{ccc}
E_{2}^{p, q}(H / K) & \stackrel{\text { Cores }_{2}}{\longrightarrow} & E_{2}^{p, q}(G / K) \\
& & \\
H^{p}\left(H / K, \mathbf{H}^{q}\left(K, M^{\bullet}\right)\right) & \stackrel{\text { Cores }}{\longrightarrow} H^{p}\left(G / K, \mathbf{H}^{q}\left(K, M^{\bullet}\right)\right)
\end{array}
$$

and such that Cores $_{i+1}$ coincides with the map induced by Cores ${ }_{i}$. This yields maps

$$
\text { Cores : } E_{\infty}^{p, q}(H / K) \rightarrow E_{\infty}^{p, q}(G / K)
$$


Moreover the corestriction maps are compatible with the filtrations on $\mathbf{H}^{p}\left(H, M^{\bullet}\right)$ and $\mathbf{H}^{p}\left(G, M^{\bullet}\right)$ and the diagram

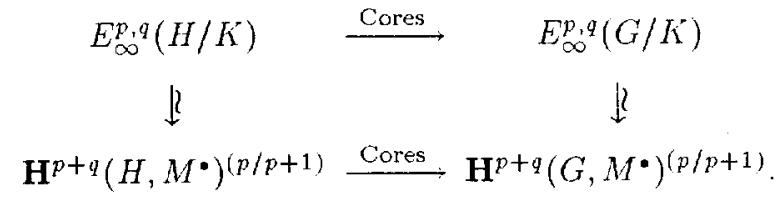

commutes.

Proof. Let us first give an explicit expression for the corestriction map

$$
H^{i}(H, M) \rightarrow H^{i}(G, M)
$$

for any $G$-module $M$. Let us fix a set-theoritic section $s: H \backslash G \rightarrow F$ which sends the class of the identity on the identity. Then it induces a map $\mathbf{Z}[G] \rightarrow \mathbf{Z}[H]$ which sends the basis element $e_{g}$ on $\epsilon_{g s(\bar{g})^{-1}}$ where $\bar{g}$ is the class of $g$ in $H \backslash G$; this map yields a map from $\mathbf{Z}[G]^{i+1}$ to $\mathbf{Z}[H]^{i+1}$. We then get a map

$$
\operatorname{Hom}_{H}\left(\mathbf{Z}[H]^{i+1}, M\right) \rightarrow \operatorname{Hom}_{H}\left(\mathbf{Z}[G]^{i+1}, M\right)
$$

and therefore a map

$$
\text { Cores : } \operatorname{Map}\left(H^{i+1}, M\right)^{H} \rightarrow \operatorname{Map}\left(G^{i+1}, M\right)^{G}
$$

such that for any $\gamma$ belonging to $\operatorname{Map}\left(H^{i+1}, M\right)^{H}$, one has

$$
\operatorname{Cores}(\gamma)\left(g_{0}, \ldots, g_{n}\right)=\sum_{x \in H \backslash G} s(x)^{-1} \gamma\left(s(x) g_{0} s\left(\overline{s(x) g_{0}}\right)^{-1}, \ldots, s(x) g_{n} s\left(\overline{s(x) g_{n}}\right)^{-1}\right) \text {. }
$$

Let now $\gamma$ belong to $C^{i}(H, M)$. The corresponding map $\tilde{\gamma}$ in Map $\left(H^{i+1}, M\right)^{H}$ is given by

$$
\tilde{\gamma}\left(h_{0}, \ldots, h_{n}\right)=h_{0} \gamma\left(h_{0}^{-1} h_{1}, h_{1}^{-1} h_{2}, \ldots, h_{n-1}^{-1} h_{n}\right)
$$

Therefore the induced map Cores : $C^{i}(H, M) \rightarrow C^{i}(G, M)$ maps $\gamma$ to

$$
\begin{aligned}
&(\text { Cores } \gamma)\left(g_{1}, \ldots, g_{n}\right) \\
&=(\operatorname{Cores} \tilde{\gamma})\left(1, g_{1}, g_{1} g_{2}, \ldots, g_{1} \ldots g_{n}\right) \\
&=\sum_{x \in H \backslash G} s(x)^{-1} \tilde{\gamma}\left(1, s(x) g_{1}\left(s\left(\overline{s(x) g_{1}}\right)\right)^{-1}, \ldots, s(x) g_{1} \ldots g_{n}\left(s\left(\overline{s(x) g_{1} \ldots g_{n}}\right)\right)^{-1}\right) \\
&=\sum_{x \in H \backslash G} s(x)^{-1} \gamma\left(s(x) g_{1}\left(s\left(\overline{s(x) g_{1}}\right)\right)^{-1},\right. \\
&\left(s(x) g_{1}\left(s\left(\overline{s(x) g_{1}}\right)\right)^{-1}\right)^{-1} s(x) g_{1} g_{2}\left(s\left(\overline{s(x) g_{1} g_{2}}\right)\right)^{-1}, \ldots \\
&\left.\left(s(x) g_{1} \ldots g_{n-1}\left(s\left(\overline{s(x) g_{1} \ldots g_{n-1}}\right)\right)^{-1}\right)^{-1} s(x) g_{1} \ldots g_{n}\left(s\left(\overline{s(x) g_{1} \ldots g_{n}}\right)\right)^{-1}\right)
\end{aligned}
$$

By construction this map $C^{i}(H, M) \rightarrow C^{i}(G, M)$ induces the corestriction map (see, for example, [Bro, chapter III, $\S 9$ (D)]).

In our case, for all $j \in \mathbf{Z}$ we get maps

$$
\text { Cores }: C^{i}\left(H, M^{j}\right) \rightarrow C^{i}\left(G, M^{j}\right) \text {. }
$$


By definition they are compatible with the coboundary operators

$$
d^{\prime}: C^{i}\left(H, M^{j}\right) \rightarrow C^{i+1}\left(H, M^{j}\right) \text { and } d^{\prime}: C^{i}\left(G, M^{j}\right) \rightarrow C^{i+1}\left(G, M^{j}\right)
$$

and it is clear that they are also compatible with the derivations $d^{\prime \prime}$ induced by the one on $M^{\bullet}$. Therefore, it only remains to check that the corestriction is compatible with the filtrations $A_{p}(H)$ and $A_{p}(G)$ defined above. So let $\gamma$ belong to $A_{l}^{(i, j)}(H)$ where $0 \leqslant l \leqslant i$. then $\gamma\left(h_{1}, \ldots, h_{i}\right)$ depends only on $h_{1}, \ldots, h_{i-l}, h_{i-l+l} K_{1} \ldots, h_{i} K$. But since $K$ is a normal subgroup of $G$ contained in $H$, the formula (4) implies that Cores $(\gamma)\left(g_{1}, \ldots, g_{i}\right)$ depends only on $g_{1}, \ldots, g_{i-l}, g_{i-l+1} K, \ldots, g_{i} K$.

\section{Proof of the main statement}

We shall decompose the proof of theorem 2.1 in a chain of lemmata. We first recall the following well known result:

Lemma 5.1. Let $V$ be a nonsingular, proper and geometrically integral variety over $k$. Let $\mathcal{G}$ be the absolute Galois group of $k$. Then there exist a canonical exact sequence

$$
0 \rightarrow \operatorname{Pic} V \rightarrow \operatorname{Pic}\left(V_{k}\right)^{\mathcal{G}} \rightarrow \mathrm{Br} k \rightarrow \mathrm{Br} k(V) .
$$

Proof. Since $V$ is nonsingular, proper and geometrically integral, we have an exact sequence

$$
0 \rightarrow k^{*} \rightarrow k(V)^{*} \rightarrow \bigoplus_{P \in V^{(1)}} \mathbf{Z} \rightarrow \operatorname{Pic} V-0
$$

as well as the corresponding one over $k^{s}$. Hilbert's theorem 90 then gives that

$$
\left(k^{s}(V)^{*} / k^{s *}\right)^{\mathcal{G}}=k(V)^{*} / k^{*}
$$

Therefore we get an exact sequence

$$
0 \rightarrow k(V)^{*} / k^{*} \rightarrow \bigoplus_{P \in V^{(1)}} \mathbf{Z} \rightarrow(\operatorname{Pic} V)^{\mathcal{G}} \rightarrow H^{1}\left(\mathcal{G}, k^{s}(V)^{*} / k^{s *}\right) \rightarrow 0
$$

and thus

$$
0 \rightarrow \operatorname{Pic} V \rightarrow(\operatorname{Pic} V)^{\mathcal{G}} \rightarrow H^{1}\left(\mathcal{G}, k^{s}(V)^{*} / k^{s *}\right) \rightarrow 0
$$

is exact. But we have also an exact sequence

$$
0 \rightarrow H^{1}\left(\mathcal{G}, k^{s}(V)^{*} / k^{s *}\right) \rightarrow H^{2}\left(\mathcal{G}, k^{s *}\right) \rightarrow H^{2}\left(\mathcal{G}, k^{s}(V)^{*}\right) .
$$

And Hilbert's theorem 90 implies that the map from $H^{2}\left(\mathcal{G}, k^{s}(V)^{*}\right)$ to $\operatorname{Br}(k(V))$ is injective.

Lemma 5.2. If $V$ is a generalized flag variety under a semi-simple linear algebraic group $G$, there exists a natural exact sequence

$$
\left(\operatorname{Pic} V^{s} \otimes k^{s *}\right)^{\mathcal{G}} \rightarrow \operatorname{Ker}\left(H^{3}(k, \mathbf{Q} / \mathbf{Z}(2)) \rightarrow H^{\mathbf{3}}(k(V), \mathbf{Q} / \mathbf{Z}(2))\right) \rightarrow \mathrm{CH}^{2}(V)_{\text {tors }} \rightarrow 0 .
$$

Proof. By [CTR, proposition 3.6], one has an exact sequence

$$
\begin{aligned}
H^{1}\left(V^{s}, \mathcal{K}_{2}\right)^{\mathcal{G}} \rightarrow H^{1}\left(\mathcal{G}, K_{2}\left(k^{s}(V)\right) / H^{0}\left(V^{s}, \mathcal{K}_{2}\right)\right) & \rightarrow \\
& \rightarrow \operatorname{Ker}\left(\mathrm{CH}^{2}(V) \rightarrow \mathrm{CH}^{2}\left(V^{s}\right)\right) \rightarrow H^{1}\left(\mathcal{G}, H^{1}\left(V^{s}, \mathcal{K}_{2}\right)\right) .
\end{aligned}
$$

By corollary $3.3, H^{1}\left(\mathcal{G}, H^{1}\left(V^{s}, \mathcal{K}_{2}\right)\right)$ is trivial and proposition 3.1 gives isomorphisms

$$
H^{0}\left(V^{s}, \mathcal{K}_{2}\right) \tilde{\rightarrow} K_{2} k^{s} \text { and } H^{1}\left(V^{s}, \mathcal{K}_{2}\right) \tilde{\rightarrow} \mathrm{Pic} V^{s} \otimes k^{s *} .
$$

Moreover, since $\mathrm{CH}^{2}\left(V^{s}\right)$ is torsion-free, one has

$$
\operatorname{Ker}\left(\mathrm{CH}^{2}(V) \rightarrow \mathrm{CH}^{2}\left(V^{s}\right)\right)=\mathrm{CH}^{2}(V)_{\text {tors }} \text {. }
$$


But by [Kah, corollaire 3.2] which is one of the key ingredient of the proof

$$
H^{1}\left(\mathcal{G}, K_{2}\left(k^{s}(V)\right) / K_{2} k^{s}\right) \stackrel{\sim}{\rightarrow} \operatorname{Ker}\left(H^{3}(k, \mathbf{Q} / \mathbf{Z}(2)) \rightarrow H^{3}(k(V), \mathbf{Q} / \mathbf{Z}(2))\right)
$$

which implies the exact sequence of the lemma.

It remains to prove that the morphism from $k_{i}^{*}$ to $H^{3}(k, \mathbf{Q} / \mathbf{Z}(2))$ is indeed the composition of a cup-product by the corestriction map. Let us first consider the case when $k_{i}=k$.

Lemma 5.3. Assume that $\mathcal{H}_{i}$ is equal to $\mathcal{G}$. Let $\alpha$ be the image of the natural generator of $\mathbf{Z}\left[\mathcal{G} / \mathcal{H}_{i}\right] \subset\left(\operatorname{Pic} V^{s}\right)^{\mathcal{G}}$ in the Brauer group of $k$. Then there is a commutative diagram

$$
\begin{aligned}
& k^{*} \longrightarrow H^{3}(k, \mathbf{Q} / \mathbf{Z}(2)) \\
& k^{*} \otimes \alpha \mathbf{Z}
\end{aligned}
$$

where the morphism at the top is the one defined in previous lemma.

Proof. The proof is exactly the same as in [Pe2, lemma 4.3], since by definition of Lichtenbaum complex $\Gamma(1)$, the morphism $\mathbf{Z} \rightarrow \operatorname{Br}(k)$ of lemma 5.1 coincides with the composition of the morphisms

$$
\mathbf{Z} \rightarrow \operatorname{Pic}\left(V^{s}\right)^{\mathcal{G}} \rightarrow H^{1}\left(\mathcal{G}, K_{1}\left(k^{s}(V)\right) / K_{1} k^{s}\right) \rightarrow \operatorname{Br}(k)
$$

where the last arrow is given by the Hochschild-Serre spectral sequence for the relative hypercohomology of the Lichtenbaum complex $\Gamma(1)$.

It remains to prove the following lemma.

Lemma 5.4. For all $i$ between 1 and $m$, one has a commutative diagram

$$
\begin{gathered}
\left(\mathbf{Z}\left[\mathcal{G} / \mathcal{H}_{i}\right] \otimes k^{s^{*}}\right)^{\mathcal{H}_{i}} \rightarrow H^{3}\left(k_{i}, \mathrm{Q} / \mathrm{Z}(2)\right) \\
N_{k_{i} / k} \downarrow \\
\left(\mathbf{Z}\left[\mathcal{G} / \mathcal{H}_{i}\right] \otimes k^{s^{*}}\right)^{\mathcal{G}} \rightarrow H^{3}(k, \mathbf{Q} / \mathbf{Z}(2)) .
\end{gathered}
$$

Proof. First let us recall the construction of the map

$$
f:\left(\operatorname{Pic}\left(V^{s}\right) \otimes k^{s *}\right)^{\mathcal{G}} \rightarrow H^{3}(k, \mathbf{Q} / \mathbf{Z}(2)) .
$$

We consider the Lichtenbaum complex $\Gamma\left(2, k^{s}\right)^{i}$ for $k^{s}$ and the one for $k(V)^{s}$ (see [Li1]. [Li2] and [Li3]). There is a canonical morphism

$$
\Gamma\left(2, k^{s}\right)^{i} \rightarrow \Gamma\left(2, k(V)^{s}\right)^{i}
$$

the cokernel of which will be denoted by $\Gamma\left(2, k(V)^{s} / k^{s}\right)^{i+1}$. Let $\mathbf{H}^{i}\left(k^{s}(V) / k^{s}, \Gamma(2)\right)$ (respectively $\mathbf{H}^{i}(k(V) / k, \Gamma(2))$ ) be the hypercohomology groups corresponding to this complex of $\operatorname{Gal}\left(k(V)^{s} / k^{s}(V)\right)$-modules (respectively Gal $\left(k(V)^{s} / k(V)\right)$-modules). Then $f$ is defined as the composition of natural morphisms

$$
\begin{aligned}
& \left(\operatorname{Pic}\left(V^{s}\right) \otimes k^{s *}\right)^{\mathcal{G}} \stackrel{f_{1}}{\longrightarrow} H^{1}\left(V^{s}, \mathcal{K}_{2}\right)^{\mathcal{G}} \stackrel{f_{2}}{\rightarrow} H^{1}\left(\mathcal{G}, K_{2}\left(k^{s}(V)\right) / K_{2}\left(k^{s}\right)\right) \stackrel{f_{3}}{\longrightarrow} \\
& \stackrel{f_{3}}{\longrightarrow} H^{1}\left(\mathcal{G}, \mathbf{H}^{3}\left(k^{s}(V) / k^{s}, \Gamma(2)\right)\right) \stackrel{f_{4}}{\longrightarrow} \mathbf{H}^{4}(k(V) / k, \Gamma(2)) \stackrel{f_{3}}{\longrightarrow} \mathbf{H}^{4}(k, \Gamma(2)) \stackrel{f_{6}}{\longrightarrow} H^{3}(k, \mathbf{Q} / \mathbf{Z}(2)) .
\end{aligned}
$$

The map $f_{2}$ is induced by the short exact sequence

$$
0 \rightarrow K_{2} k^{s}(V) / K_{2} k^{s} \rightarrow \mathcal{Z} \rightarrow H^{1}\left(V^{s}, \mathcal{K}_{2}\right) \rightarrow 0
$$


where $\mathcal{Z}$ is the kernel of the map

$$
\bigoplus_{x \in V^{s(1)}} k^{s}(x)^{*} \rightarrow \bigoplus_{x \in V^{s(2)}} \mathrm{Z} .
$$

The morphism $f_{3}$ is induced by the natural morphism

$$
\mathbf{H}^{2}\left(k^{s}(V), \Gamma(2)\right) \rightarrow \mathbf{H}^{3}\left(k^{s}(V) / k^{s}, \Gamma(2)\right)
$$

and the isomorphism from $\mathbf{H}^{2}\left(k^{s}(V), \Gamma(2)\right)$ to $K_{2} k^{s}(V), f_{4}$ by the spectral sequence

$$
H^{p}\left(\mathcal{G}, \mathbf{H}^{q}\left(k^{s}(V) / k^{s}, \Gamma(2)\right)\right) \Rightarrow \mathbf{H}^{p+q}(k(V) / k, \Gamma(2))
$$

and $f_{6}$ by the canonical isomorphism

$$
f_{7}: H^{3}(k, \mathbf{Q} / \mathbf{Z}(2)) \sim \mathbf{H}^{4}(k, \Gamma(2)) .
$$

The maps $f_{1}, f_{2}$ and $f_{3}$ are clearly compatible with the corestriction map. The map $f_{7}$ is induced by a morphism of complexes of Galois modules whereas the map $f_{5}$ is a cobordism for a short sequence of complexes. Therefore the maps $f_{5}$ and $f_{6}$ are compatible with the corestriction. Finally the compatibility of $f_{4}$ with the corestriction is a consequence of lemma 4.2 .

\section{Connection with Panin's result}

Let us first recall this result which yields the $K$-theory groups of the variety $V$.

Theorem 6.1 (Panin, $[\mathrm{Pa}]$ ). If $V$ is a generalized flag variety over a field $k$ then there exists a natural separable algebra $A$ over $k$ and an isomorphism

$$
K_{*} X \stackrel{\sim}{\rightarrow} K_{*} A \text {. }
$$

Proposition 6.2. Assume that the field $k$ is perfect or that the center of $G$ is a reduced $k$-group. With notation as in the preceding theorem and theorem 2.1 , there exists a natural decomposition of $A$ in the product of two separable algebras $C$ and $D$ such that one has $C \stackrel{\sim}{\rightarrow} \times_{i=1}^{m} C_{i}$ where $C_{i}$ is a simple central algebra of centre $k_{i}$, the class of which is equal to $\alpha_{i}$ in $\mathrm{Br} k_{i}$.

Remark 6.3. The decomposition is given explicitly in the proof.

Proof. We use the notation of section 3. We shall first recall Panin's construction of the algebra $A$. There exists a simply connected quasi-split $k$-form $\tilde{G}^{q}$ of $G$, a parabolic subgroup $\tilde{P}^{q}$ of $\tilde{G}^{q}$ and an element $\gamma$ of $H^{1}\left(k, G^{q}\right)$ where $G^{q}=\tilde{G}^{q} / Z\left(\tilde{G}^{q}\right)$ such that $V$ is the twisted $k$-form of $\tilde{P}^{q} \backslash \tilde{G}^{q}$ defined by $\gamma$ and $G$ the corresponding $k$-form of $\tilde{G}^{q}$. Let $\tilde{B}^{q}$ be a Borel subgroup of $\tilde{G}^{q}$ defined over $k$ and contained in $\tilde{P}^{q}$ and $\tilde{T}^{q}$ be a maximal torus of $\tilde{B}^{q}$. We may assume that the isomorphism $G^{s} \sim\left(\tilde{G}^{q}\right)^{s}$ sends $\left(\tilde{P}^{q}\right)^{s}$ (resp. $\left(\tilde{B}^{q}\right)^{s}$, $\left.\left(\tilde{T}^{q}\right)^{s}\right)$ on $P$ (resp. $B, T$ ). We denote by $U_{w}^{\prime q}$ the images of $U_{w}^{\prime}$ in $\left(\tilde{G}^{q}\right)^{s}$.

By [St], the ring of representations $R(P)$ of $P$ over $\bar{k}$ has a canonical basis as a module over $R(G \times \bar{k})$ which is defined as follows: let $W^{I^{\prime}}$ be the set

$$
\left\{w \in W \mid \forall \alpha \in I, w \alpha \in \Phi^{+}\right\}
$$

then the basis $\left(e_{w}^{\prime}\right)_{w \in W^{I}}$ is given by:

$$
e_{w}^{\prime}=\sum_{\lambda \in W_{I} \cdot \lambda_{w}} \lambda \in \mathbf{Z}\left[X^{*}(T)\right]^{W_{I}} \simeq R(P)
$$

where

$$
\lambda_{w}=\sum_{\left\{\alpha \in \Delta \mid w^{-1} \alpha<0\right\}} w^{-1} \varpi_{\alpha} \in X^{*}(T) .
$$


The set $W^{I^{\prime}}$ is globally invariant under the natural action of $\mathcal{G}$ on $W$. Let $E$ be the commutative separable algebra corresponding to the $\mathcal{G}$-set $W^{I^{\prime}}$. The basis $\left(e_{w}^{\prime}\right)_{w} \in W^{\prime \prime}$ defines a representation of $P^{q}$ over $E$. Let $V$ be the induced representation from $P^{q}$ to $G^{q}, A^{q}$ the ring End $E(V)$ and $A_{\gamma}^{q}$ the twisted form of $A^{q}$ by $\gamma$. The algebra $A=A_{\gamma}^{q}$ is the algebra constructed by Panin in $[\mathrm{Pa}, \S 12]$.

Lemma 6.4. The set $W^{I^{\prime}}$ coincides with $\left(W^{I}\right)^{-1}$.

Proof. Let $w$ belong to $W^{I}$. Assume that $w^{-1}$ does not belong to $W^{I^{\prime}}$. Let $\alpha \in I$ be such that $w^{-1} \alpha<0$. Then by [Bki, chapitre VI, $\mathrm{n}^{\circ} 1.6$, pages $157-158$, corollaires 1 et 2]

$$
l\left(w^{-1} s_{\alpha}\right)=\#\left\{\beta \in \Phi^{+} \mid w^{-1} s_{\alpha} \beta<0\right\}=l\left(w^{-1}\right)-1 .
$$

Then $w$ is not of minimal length in its class $\bar{w} \in W_{I} \backslash W$ which is in contradiction with the hypothesis. thus $\left(W^{I}\right)^{-1}$ is a subset of $W^{I^{\prime}}$. But $W^{I^{\prime}}$ is a set of representing elements for $W / W^{I}$ (see [St, lemma 2.5(a)]) and both sets have the same cardinal.

In the sequel we put $e_{w}=e_{w-1}^{\prime}$ for all $w$ in $W^{I}$.

Lemma 6.5. For any $\alpha$ in $\Delta-I$ one has that $w_{\Delta-\{\alpha\}} w_{\Delta}$ belongs to $W^{I}$ and

$$
e_{w_{\Delta-\{\alpha\}} w_{\Delta}}=-\varpi_{\alpha} \text {. }
$$

Proof. Since $w_{\Delta-\{\alpha\}} w_{\Delta}$ is of minimal length in its class modulo $W_{\Delta-\{\alpha\}}$, it is a fortiori of minimal length modulo $W_{I}$. Moreover

$$
\left\{\beta \in \Delta \mid w_{\Delta-\{\alpha\}} w_{\Delta} \beta<0\right\}=\left\{\beta \in \Delta \mid w_{\Delta-\{\alpha\}}{ }^{\epsilon} \beta>0\right\}=\left\{{ }^{\epsilon} \alpha\right\}
$$

where $\epsilon: \Delta \rightarrow \Delta$ is the involutive bijection such that $w_{\Delta} \cdot \alpha=-{ }^{\epsilon} \alpha$. Thus we get

$$
\lambda_{w_{\Delta-\{\alpha\}} w_{\Delta}}=w_{\Delta-\{\alpha\}} w_{\Delta} \varpi_{\epsilon_{\alpha}}=w_{\Delta-\{\alpha\}} \cdot\left(-\varpi_{\alpha}\right)=-\varpi_{\alpha} .
$$

But $-\varpi_{\alpha}$ is invariant under $W_{I}$ and the lemma is proved.

End of the proof of proposition 6.2. Let $C$ be the algebra constructed from the $\mathcal{G}$-set $\left(e_{w}\right)_{w \in\left\{w_{\Delta-\{\alpha\}} w_{\Delta}, \alpha \in \Delta-I\right\}}$. Then one may write $A$ as $C \times D$ where $D$ corresponds to the complementary set. The above $\mathcal{G}$-set is canonically isomorphic to the $\mathcal{G}$-set $\left(-\varpi_{\alpha}\right)_{\alpha \in \Delta-I}$. Thus we get a canonical isomorphism of étale algebras

$$
Z(C) \stackrel{\sim}{\rightarrow}\left(\operatorname{Pic} V^{s} \otimes k^{s}\right)^{\mathcal{G}} .
$$

Let $E$ be this algebra. Let $\chi$ be the natural character $Z\left(\tilde{G}^{q}\right) \rightarrow \mathbf{G}_{m E}$ defined by $\left(-\varpi_{\alpha}\right)_{\alpha \in \Delta-I}$. Let $\eta_{1}$ be the image of $\gamma$ by the composite map

$$
H^{1}\left(k, G^{q}\right) \rightarrow H^{1}\left(E, G^{q}\right) \stackrel{\partial}{\rightarrow} H^{2}\left(E, Z\left(\tilde{G}^{q}\right)\right) \stackrel{x^{*}}{\rightarrow} H^{2}\left(E, \mathbf{G}_{m}\right) .
$$

Then, thanks to the hypothesis on $k$, the components of $\eta_{1}$ in the decomposition

$$
H^{2}\left(E, \mathbf{G}_{m}\right) \tilde{\rightarrow} \prod_{i=1}^{m} \operatorname{Br} k_{i}
$$

are the classes of the algebras $B_{i}$ (see [Pa, lemma 3.3] and [Ti, 4.2]). For any $\mathcal{G}$-module $M, \tilde{M}$ denotes the corresponding étale sheaf on Spec $k$. By definition of the fields $k_{i}$ there is an isomorphism from $H^{0}\left(E, \widetilde{\operatorname{Pic} V^{s}}\right)$ onto $\bigoplus_{1 \leqslant i, j \leqslant m} H^{0}\left(k_{i}, \mathbf{Z}\left[\mathcal{G} / \mathcal{H}_{j}\right]\right)$. Let $\eta_{2}$ be the image of the sum of the elements $\mathcal{H}_{i}$ of $\mathbf{Z}\left[\mathcal{G} / \mathcal{H}_{i}\right]^{\mathcal{H}_{i}}$ by the composite morphism

$$
\bigoplus_{1 \leqslant i \leqslant m} H^{0}\left(k_{i}, \mathbf{Z}\left[\mathcal{G} / \mathcal{H}_{i}\right]\right) \hookrightarrow H^{0}\left(E, \widetilde{\operatorname{Pic} V^{s}}\right) \stackrel{\partial_{1}}{\longrightarrow} H^{1}\left(E, k^{s}\left(\widetilde{V)^{*} / k^{s^{*}}}\right) \stackrel{\partial_{2}}{\longrightarrow} H^{2}\left(E, \mathbf{G}_{m}\right)\right.
$$


where $\partial_{1}$ is the coboundary homomorphism for the short exact sequence

$$
0 \rightarrow k^{s}(V)^{*} / k^{s *} \rightarrow \operatorname{Div} V^{s} \rightarrow \operatorname{Pic} V^{s} \rightarrow 0
$$

and $\partial_{2}$ the coboundary homomorphism for the short exact sequence

$$
0 \rightarrow k^{s *} \rightarrow k^{s}(V)^{*} \rightarrow k^{s}(V)^{*} / k^{s *} \rightarrow 0 .
$$

Then, by definition, the $\alpha_{i}$ are the components of $\eta_{2}$. Thus it remains to prove the following lemma:

Lemma 6.6. With notation as above, the classes $\eta_{1}$ and $\eta_{2}$ coincide.

Proof. Let us fix $i$ in $\{1, \ldots, m\}$. It is enough to prove that the image $\xi_{2}$ of the generator of $\mathbf{Z}\left[\mathcal{G} / \mathcal{H}_{i}\right]^{\mathcal{H}_{i}}$ by the composite map

$$
\mathrm{Z}\left[\mathcal{G} / \mathcal{H}_{i}\right]^{\mathcal{H}_{\imath}} \hookrightarrow H^{0}\left(k_{i}, \operatorname{Pic} V^{s}\right) \rightarrow H^{1}\left(k_{i}, k^{s}(V)^{*} / k^{s *}\right) \rightarrow H^{2}\left(k_{i}, k^{s *}\right)
$$

coincides with the component of $\eta_{1}$ in $\operatorname{Br} k_{i}$ which we denote by $\xi_{1}$. Let $C$ be the orbit of $\mathcal{G}$ in $\Delta-I$ corresponding to $k_{i}$ and $\chi_{i}: Z\left(\tilde{G}^{q}\right) \rightarrow \mathbf{G}_{m k_{i}}$ be the corresponding character. We denote also by

$$
\gamma: \mathcal{G} \rightarrow G^{q}\left(k^{s}\right)
$$

a cocycle which represents $\gamma$ so that for any $\sigma$ in $\mathcal{G}$ the composite map

$$
G^{q} \times k^{s} \stackrel{\operatorname{Id} \times \sigma^{-1}}{\longrightarrow} G^{q} \times k^{s} \tilde{\rightarrow} G \times k^{s} \stackrel{\operatorname{Id} \times \sigma}{\longrightarrow} G \times k^{s} \stackrel{\sim}{\rightarrow} G^{q} \times k^{s}
$$

coincides with the interior automorphism Int $\gamma(\sigma)$. Let $x \mapsto \tilde{x}$ be a set-theoritic section of the canonical map

$$
\tilde{G}^{q}\left(k^{s}\right) \rightarrow G^{q}\left(k^{s}\right)
$$

which is surjective, by the hypothesis on $k$. Then $\xi_{1}$ is the image by $\chi$ of the cocycle

$$
\begin{aligned}
\tilde{\xi}_{1}: \mathcal{G}^{2} & \rightarrow Z\left(G^{q}\right)\left(k^{s}\right) \\
\left(\sigma_{1}, \sigma_{2}\right) & \mapsto{ }^{\sigma_{1}} \widetilde{\gamma\left(\sigma_{2}\right)} \widehat{\gamma\left(\sigma_{1} \sigma_{2}\right)}-1 \widetilde{\gamma\left(\sigma_{1}\right) .}
\end{aligned}
$$

Let $w_{\Delta}^{\prime}$ be a lifting of $w_{\Delta}$ in $\mathcal{N}_{\tilde{G} q}\left(\tilde{T}^{q}\right)$. Let $s_{1}:$ Pic $V^{s} \rightarrow \operatorname{Div} V^{s}$ be the section which sends the class of $\overline{\pi(B w B)}$ to $\overline{\pi(B w B)}$ for $w \in W_{\operatorname{dim} V-1}^{I}$. For all $\alpha \in \Delta-I$ let $f_{\alpha}$ be the function on $\tilde{G}^{q}$ defined by

$$
\forall b \in \tilde{B}^{q}\left(k^{s}\right), \forall u \in U_{w_{\Delta}^{\prime}}^{\prime q}\left(k^{s}\right), f_{\alpha}\left(b w_{\Delta} u\right)=\varpi_{\alpha}(b)^{-1} .
$$

By [Pe3, page 164] this function induces the section of the sheaf defined by $\varpi_{\alpha}$ which corresponds to the divisor $\overline{\pi\left(B^{q} \bar{s}_{\alpha} B^{q}\right)}$. The stabilizer of $\overline{\pi\left(B^{q} \bar{s}_{\alpha} B^{q}\right)} \subset P^{q} \backslash G^{q}$ contains $B$. It is thus a standard parabolic subgroup of $G^{q}$. But for any $\beta \in \Delta$ one has:

$$
\begin{aligned}
\overline{\pi\left(B^{q} \bar{s}_{\alpha} B^{q}\right)} s_{\beta} & \subset \overline{\pi\left(B^{q} \tilde{s}_{\alpha} B^{q}\right)} \\
\Leftrightarrow B^{q} s_{\alpha} w_{\Delta} B^{q} s_{\beta} & \subset \overline{B^{q} s_{\alpha} w_{\Delta} B^{q}} \\
\Leftrightarrow B^{q} w_{\Delta} s_{\alpha} B^{q} s_{\beta} & \subset \overline{B^{q} w_{\Delta} s_{\alpha} B^{q}} \\
\Leftrightarrow l\left(w_{\Delta} s_{\epsilon_{\alpha}} s_{\beta}\right) & =l\left(w_{\Delta} s_{\alpha}\right)-1 \\
\Leftrightarrow \beta & \in \Delta-{ }^{\epsilon} \alpha .
\end{aligned}
$$

where the third equivalence follows from the fact that, by [Bki, chapitre IV, $\S 2,\left(3^{\prime}\right)$ et théorème 2],

$$
\operatorname{dim}\left(B^{q} w_{\Delta} s_{\epsilon_{\alpha}} B^{q} s_{\beta} B^{q}\right)=\operatorname{dim} B^{q}+\sup \left(l\left(w_{\Delta} s_{\epsilon_{\alpha}} s_{\beta}\right), l\left(w_{\Delta} s_{\epsilon_{\alpha}}\right)\right) .
$$


We then choose a section

$$
s: P_{\Delta-{ }_{\alpha}}^{q} \backslash G^{q}\left(k^{s}\right) \rightarrow G^{q}\left(k^{s}\right) .
$$

Then we may choose a section $s_{2}$

$$
s_{2}: k^{s}(V)^{*} / k^{*} \rightarrow k^{s}(V)^{*}
$$

such that for any $g \in \tilde{G}^{q}\left(k^{s}\right)$ one has

$$
s_{2}\left(-\overline{\pi\left(B^{q} \bar{s}_{\alpha} B^{q}\right)}+\overline{\pi\left(B^{q} \vec{s}_{\alpha} B^{q}\right)} \cdot g^{-1}\right)(h)=f_{\alpha}(h)^{-1} f_{\alpha}(\widehat{h s(\hat{g})})
$$

where $\hat{g}$ is the class of $g$ in $P_{\Delta-{ }^{\epsilon} \alpha}^{q} \backslash G^{q}\left(k^{s}\right)$. We are now able to compute

$$
\xi_{2}=\partial_{2} \circ \partial_{1}\left(\left[\overline{\pi\left(B^{q} \bar{s}_{\alpha} B^{q}\right)}\right]\right) .
$$

First, using $s_{1}$, the class $\partial_{1}\left(\left[\overline{\pi\left(B^{q} \bar{s}_{\alpha} B^{q}\right)}\right]\right)$ is represented by the cocycle

$$
\sigma \mapsto-\overline{\pi\left(B^{q} \bar{s}_{\alpha} B^{q}\right)}+\overline{\pi\left(B^{q} \bar{s}_{\alpha} B^{q}\right)} \cdot \gamma(\sigma)^{-1} \in \operatorname{Ker}\left(\operatorname{Div}\left(V^{s}\right) \rightarrow \operatorname{Pic}\left(V^{s}\right)\right)
$$

Let $\tilde{\gamma}$ (resp. $\tilde{s}$ ) be the composite of $\gamma$ (resp. $s$ ) with the section $x \mapsto \tilde{x}$. Then $\xi_{2}$ is represented by the cocycle

$$
\begin{aligned}
\left(\sigma_{1}, \sigma_{2}\right) \mapsto[g \mapsto & f_{\alpha}\left(g \tilde{\gamma}\left(\sigma_{1}\right)\right)^{-1} f_{\alpha}\left(g^{\sigma_{1}} \tilde{s}\left(\widehat{\gamma\left(\sigma_{2}\right)}\right) \tilde{\gamma}\left(\sigma_{1}\right)\right) \\
& \left.. f_{\alpha}(g) f_{\alpha}\left(g \tilde{s}\left(\widehat{\gamma\left(\sigma_{1} \sigma_{2}\right.}\right)\right)\right)^{-1} \\
& \left.f_{\alpha}(g)^{-1} f_{\alpha}\left(g \tilde{s}\left(\widehat{\gamma\left(\sigma_{1}\right)}\right)\right)\right]
\end{aligned}
$$

But for any $g$ in $\tilde{G}^{q}\left(k^{s}\right), g^{-1} \tilde{s}(\hat{g})$ belongs to $P_{\Delta-\epsilon_{\alpha}}\left(k^{s}\right)$ and for any $p \in P_{\Delta-{ }^{e} \alpha}\left(k^{s}\right)$, the expression $\varpi_{\alpha}\left(w_{\Delta} p w_{\Delta}\right)$ is well defined and

$$
\forall g \in \tilde{G}^{q}\left(k^{s}\right), f_{\alpha}(g p)=\varpi_{\alpha}\left(w_{\Delta} p w_{\Delta}\right)^{-1} f_{\alpha}(g) .
$$

We get that $\xi_{2}$ is represented by the cocycle

$$
\begin{aligned}
\left(\sigma_{1}, \sigma_{2}\right) \mapsto[g \mapsto & f_{\alpha}\left(g \tilde{\gamma}\left(\sigma_{1}\right)\right)^{-1} f_{\alpha}\left(g^{\sigma_{1}} \tilde{\gamma}\left(\sigma_{2}\right) \tilde{\gamma}\left(\sigma_{1}\right)\right) \\
& f_{\alpha}\left(g \tilde{\gamma}\left(\sigma_{1} \sigma_{2}\right)\right)^{-1} f_{\alpha}\left(g \tilde{\gamma}\left(\sigma_{1}\right)\right) \\
& .\left({ }^{\sigma_{1}} \varpi_{\alpha}\left(w_{\Delta} \tilde{\gamma}\left(\sigma_{2}\right)^{-1} \tilde{s}\left(\widehat{\gamma\left(\sigma_{2}\right)}\right) w_{\Delta}\right)^{-1}\right. \\
& \left.. \varpi_{\alpha}\left(w_{\Delta} \tilde{\gamma}\left(\sigma_{1} \sigma_{2}\right)^{-1} \tilde{s}\left(\widehat{\gamma\left(\sigma_{1} \sigma_{2}\right.}\right)\right) w_{\Delta}\right) \\
& \left.\left.\varpi_{\alpha}\left(w_{\Delta} \tilde{\gamma}\left(\sigma_{1}\right)^{-1} \tilde{s}\left(\widehat{\gamma\left(\sigma_{1}\right)}\right) w_{\Delta}\right)^{-1}\right)\right]
\end{aligned}
$$

Then removing a coboundary and using $(6)$ one gets that $\xi_{2}$ is represented by

$$
\left(\sigma_{1}, \sigma_{2}\right) \mapsto-\varpi_{\alpha}\left(\tilde{\xi}_{1}\left(\sigma_{1}, \sigma_{2}\right)\right)
$$




\section{A FEW EXAMPLES}

7.1. The low-dimensional cases.

Corollary 7.1. With the notation of theorem 2.1, the complex $\mathcal{C}$ is exact if $\operatorname{dim} V \leqslant 2$.

Proof. In this case $K_{0}\left(V^{s}\right)^{3}=\{0\}$. Thus, by proposition $3.4(\mathrm{v}), \mathrm{CH}^{2}(V)_{\text {tors }}$ is trivial. The corollary is then a direct consequence of theorem 2.1 .

Corollary 7.2. If $\operatorname{dim} V=3$ then the homology of the complex $\mathcal{C}$ is either cyclic or trivial.

Proof. In this case $K_{0}\left(V^{s}\right)^{3}=\mathrm{Z}$. Thus proposition $3.4(\mathrm{v})$ and theorem 2.1 imply the result.

Remark 7.3. The paragraph 6.2 of [Pe2] gives an example for which $\operatorname{dim} V=3$ and $(\mathcal{C})$ is not exact.

7.2. The case of orthogonal groups. We shall first give an explicit description of the complex $(\mathcal{C})$ in the case of a flag variety under an orthogonal group.

Let $k$ be a field of characteristic different from 2. Let $q$ be a nondegenerate quadratic form of dimension $n$ over $k$. We assume that $n$ is bigger than 3 . Let $G$ be the group $\operatorname{PSO}(q)$ and $V$ be a flag variety under $G$.

By [MPW, proposition 1.3], these varieties are characterized by the form $q$ and their type over $k^{s}$. We recall their description (see [MPW, \$5]). If $n$ is odd, $n=2 m+1$ then $G$ is of type $B_{m}$. The root system is given in $\mathbf{R}^{m}$ by the basis $\alpha_{i}=\epsilon_{i}-\epsilon_{i+1}$ if $1 \leqslant i \leqslant m-1$ and $\alpha_{m}=\epsilon_{m}$ where $\left(\epsilon_{i}\right)_{1 \leqslant i \leqslant m}$ is the standard basis of $\mathbf{R}^{m}$. The flag variety $X\left(q, n_{1}, \ldots, n_{l}\right)$ corresponding to $\Delta-\left\{\alpha_{n_{1}}, \ldots, \alpha_{n_{l}}\right\}$ with $1 \leqslant n_{1}<\cdots<n_{l} \leqslant m$ is such that

$$
\begin{aligned}
X\left(q, n_{1}, \ldots, n_{l}\right)\left(k^{s}\right)=\{ & \left(W_{1}, \ldots, W_{l}\right) \mid W_{1} \subset \cdots \subset W_{l} \subset V \otimes k^{s}, \\
& \left.W_{l} \text { is totally isotropic and } \operatorname{dim}_{K} W_{i}=n_{i}\right\}
\end{aligned}
$$

as a set with Galois action.

If $n$ is even, $n=2 m$, then $G$ is of type $D_{m}$, the root system is given in $\mathbf{R}^{m}$ by the basis $\alpha_{i}=\epsilon_{i}-\epsilon_{i+1}$ if $1 \leqslant i \leqslant m-1$ and $\alpha_{m}=\epsilon_{m-1}+\epsilon_{m}$.

If the signed discriminant of $q, d_{ \pm} q$, is a square then we are in the inner case. The variety of maximal totally isotropic spaces has two components. Over $k^{s}$, the form $q$ may be written as $\sum_{i=1}^{m} x_{i} x_{2 m-i}$ over a basis $\left(e_{i}\right)_{1 \leqslant i \leqslant 2 m}$. We choose the maximal torus to be the diagonal matrices $D\left(\alpha_{1}, \ldots, \alpha_{m}, \alpha_{m_{2}}^{-1}, \ldots, \alpha_{1}^{-1}\right)$ and $\epsilon_{i}$ sends this diagonal element on $\alpha_{i}$. Let us denote by $\mathcal{M}^{+}$the component containing $\left\langle e_{1}, \ldots, e_{n}\right\rangle$ and by $\mathcal{M}^{-}$the other one. If $1 \leqslant n_{1}<\cdots<n_{l} \leqslant m-1$ then $X\left(q, n_{1}, \ldots, n_{l}\right)$ is the flag variety such that

$$
X\left(q, n_{1}, \ldots, n_{l}\right)\left(k^{s}\right)=\left\{\left(W_{1}, \ldots, W_{l}\right) \mid W_{1} \subset \cdots \subset W_{l} \subset V \otimes k^{s}\right.
$$

$W_{I}$ is totally isotropic and $\left.\operatorname{dim}_{k} s W_{i}=n_{i}\right\}$.

If $n_{l} \leqslant m-2$ then the corresponding set of roots is $\Delta-\left\{\alpha_{n_{1}}, \ldots, \alpha_{n_{l}}\right\}$; if $n_{l}=m-1$ then the corresponding set is $\Delta-\left\{\alpha_{n_{1}}, \ldots, \alpha_{n_{l-1}}, \alpha_{m-1}, \alpha_{m}\right\}$ If $1 \leqslant n_{1}<\cdots<n_{l-1} \leqslant m-2$ and $n_{l}=m$, then

$$
X^{+}\left(q, n_{1}, \ldots, n_{l}\right)\left(k^{s}\right)=\left\{\left(W_{1}, \ldots, W_{l}\right) \mid W_{1} \subset \cdots \subset W_{l} \subset V \otimes k^{s},\right.
$$

$W_{l}$ is totally isotropic, $\operatorname{dim}_{k^{s}} W_{i}=n_{i}$ and $\left.W_{l} \in \mathcal{M}^{+}\left(k^{s}\right)\right\}$.

Similarly, one defines $X^{-}\left(q, n_{1}, \ldots, n_{l}\right)$. The first variety corresponds to the set $\Delta-$ $\left\{\alpha_{n_{1}}, \ldots, \alpha_{n_{l-1}}, \alpha_{n}\right\}$ and the second one to $\Delta-\left\{\alpha_{n_{1}}, \ldots, \alpha_{n_{l-1}}, \alpha_{n-1}\right\}$. 
If $d_{ \pm} q$ is not a square we are in the outer case. Let $L$ be the field $k\left(\sqrt{d_{ \pm} q}\right)$. The absolute Galois group of $k$ acts through $\mathrm{Gal}(L / k)$ on the system of roots. If $1 \leqslant n_{1}<$ $\cdots<n_{l} \leqslant m-1$ the variety $X\left(q, n_{1}, \ldots, n_{l}\right)$ is defined as in the previous case. The set of roots is $\Delta-\left\{\alpha_{n_{1}}, \ldots, \alpha_{n_{l}}\right\}$ if $n_{l} \neq m-1$ and $\Delta-\left\{\alpha_{n_{1}}, \ldots, \alpha_{n_{l-1}}, \alpha_{n-1}, \alpha_{n}\right\}$ otherwise.

Lemma 7.4. Let $V$ be one of the flag varieties described above. Let $I$ be the corresponding set of roots. The non-trivial component of the algebra $C$ given by the proposition 6.2 is similar to

(i) $C_{0}(q)$ if $n=2 m+1$ and $\alpha_{m} \in \Delta-I$,

(ii) $C_{0}(q)$ if $n=2 m$ and $\left\{\alpha_{m}, \alpha_{m-1}\right\} \subset \Delta-I$,

(iii) $C_{+}(q)$ if $n=2 m, d_{ \pm} q \in k^{* 2}$ and $\alpha_{m}$ or $\alpha_{m-1}$ does not belong to $I$

In all other cases $C$ is a product of trivial algebras.

Remark 7.5. This result generalizes easily to the case of a central simple algebra of even degree with an involution of the first kind and of orthogonal type.

Proof. The center of the algebra $C$ is the étale algebra corresponding to the Galois set $\left\{\varpi_{\alpha}\right\}$ for $\alpha \in \Delta-I$. It is non-trivial only when $n=2 m, d_{ \pm} q \notin k^{* 2}$ and $\left\{\alpha_{m}, \alpha_{m-1}\right\} \subset$ $\Delta-1$ in which case it is $k\left(\sqrt{d_{ \pm} q}\right)$. By construction, (see $\left.(5)\right)$ the classes of the components of $C$ depend only on the restriction of the characters $\varpi_{\alpha}$ to the center $\mathcal{Z}$ of $\operatorname{Spin}(q)$ for $\alpha \in \Delta-I$. By [Bki, planches II et IV], these restrictions are as follows:

- If $n=2 m+1$, then $\mathcal{Z}=\mathbf{Z} / 2 \mathbf{Z}$ and $\varpi_{i \mid \mathcal{Z}}$ generates $\operatorname{Hom}\left(\mathcal{Z}, \mathbf{G}_{m}\right)$ if and only if $i=m$.

- If $n=2 m$ with $m$ even and $d_{ \pm} q \in k^{* 2}$ then $\operatorname{Hom}\left(\mathcal{Z}, \mathbf{G}_{m}\right) \stackrel{\sim}{\rightarrow} \mathbf{Z} / 2 \mathbf{Z} \times \mathbf{Z} / 2 \mathbf{Z}$ so that the restriction of $\varpi_{i}$ with $i<l-1$ is trivial if $i$ is even and $(1,1)$ if $i$ is odd, the restriction of $\varpi_{l-1}$ corresponds to $(1,0)$ and the one of $\varpi_{l}$ to $(0,1)$.

- if $n=2 m$ with $m$ odd and $d_{ \pm} q \in k^{* 2}$ then $\operatorname{Hom}\left(\mathcal{Z}, \mathbf{G}_{m}\right) \stackrel{\sim}{\rightarrow} \mathbf{Z} / 4 \mathbf{Z}$ so that the restrictions of $\varpi_{i}$ with $i<l-1$ is trivial if $i$ is even and equal to 2 if $i$ is odd, the restriction of $\varpi_{l-1}$ is 1 and the one of $\varpi_{l} 3$.

- If $n=2 m$ and $d_{ \pm} q \notin k^{* 2}$, then the above description is valid over $L=k\left(\sqrt{d_{ \pm} q}\right)$ and is compatible with the action of $\mathrm{Gal}(L / k)$ over $\Delta-I$ and $\mathbf{Z} / 4 \mathbf{Z}$ or $\mathbf{Z} / 2 \mathbf{Z} \times$ $\mathbf{Z} / 2 \mathbf{Z}$.

But, by [MPW, Proposition 2.2], the class of a component corresponding to a morphism $\phi$ from $\mathcal{Z}$ to $\mathbf{G}_{m, E}$ is given by the class of any central algebra $A$ over $E$ such that there exists a representation from $\operatorname{Spin}(q)_{E}$ to $\mathrm{GL}_{1}(A)$ which extends $\phi$.

If $n$ is odd the natural injection Spin $(q) \rightarrow \mathrm{GL}\left(C_{0}(q)\right)$ restricts itself to the generator of $\operatorname{Hom}\left(\mathcal{Z}, \mathbf{G}_{m}\right)$ and we get $(i)$.

If $n$ is even, $n=2 m$, and $d_{ \pm} q \in k^{* 2}$ then the injection

$$
\operatorname{Spin}(q) \hookrightarrow C_{0}(q) \stackrel{\sim}{\rightarrow} C_{+}(q) \times C_{-}^{\prime}(q)
$$

gives, by projection, the generators of $\operatorname{Hom}\left(\mathcal{Z}, \mathbf{G}_{m}\right)$. This implies (ii) and (iii).

In the case of quadrics, the results of Karpenko on the torsion subgroup in the second Chow group enables us to give a slight refinement of Arason's results. 'This refinement seems to be known but we give it as an illustration of our results.

For any $\left(a_{1}, \ldots, a_{n}\right)$ in $k^{* n}$ the $n$-Pfister form $\ll a_{1}, \ldots, a_{n} \gg$ is the form

$$
<1,-a_{1}>\otimes \cdots \otimes<1,-a_{n}>\text {. }
$$

A quadratic form $q$ of dimension $n$ is said to be a neighbour of a $r$-Pfister form $q^{\prime}$ if and only if $n>2^{r-1}$ and $q$ is similar to a subform of $q^{\prime}$. 
Proposition 7.6. Let $q$ be an anisotropic quadratic form of dimension bigger than three and let $Q$ be the corresponding projective quadric then the following cases are possible:

(i) (Arason [Ara, Satz 5.4]) If $q$ is a neighbour of a 2-Pfister form $\ll a, b \gg$ then the sequence

$$
H^{1}(k, \mathbf{Z} / 2 \mathbf{Z}) \stackrel{\cup(a, b)}{\longrightarrow} H^{3}(k, \mathbf{Z} / 2 \mathbf{Z}) \rightarrow H^{3}(k(Q), \mathbf{Z} / 2 \mathbf{Z})
$$

is exact.

(ii) If the dimension of $q$ is four and $q$ is not similar to a 2-Pfister form, let $d$ be its discriminant and $(a, b)$ its Pfister invariant. Then the sequence

$$
H^{1}(k(\sqrt{d}), \mathbf{Z} / 2 \mathbf{Z}) \stackrel{N(.) \cup(a, b)}{\longrightarrow} H^{3}(k, \mathbf{Z} / 2 \mathbf{Z}) \rightarrow H^{3}(k(Q), \mathbf{Z} / 2 \mathbf{Z})
$$

is exact.

(iii) (Arason [Ara, Satz 5.6]) If the form is a neighbour of 3 -pfister form $\ll a, b, c \gg$ then $\operatorname{Ker}\left(H^{3}(k, \mathbf{Z} / 2 \mathbf{Z}) \rightarrow H^{3}(k(Q), \mathbf{Z} / 2 \mathbf{Z})\right)$ is the subgroup generated by $(a, b, c)$.

(iv) (See [Ara, page 469]) In all other cases this kernel is trivial.

Remark 7.7. For assertion (iii), theorem 2.1 and the result of Karpenko implies only that the kernel is isomorphic to $\mathbf{Z} / 2 \mathbf{Z}$.

Proof. We only prove assertions (i), (ii) and (iv). By [Kar, theorem 6.1] the torsion subgroup of $\mathrm{CH}^{2}(Q)$ is trivial except when $q$ is anisotropic and the neighbour of a 3 -fold Pfister form in which case this group is isomorphic to $\mathbf{Z} / 2 \mathbf{Z}$. Here we have

$$
\Delta-I= \begin{cases}\left\{\alpha_{1}, \alpha_{2}\right\} & \text { if } n=4 \\ \left\{\alpha_{1}\right\} & \text { otherwise }\end{cases}
$$

Thus, by lemma 7.4 , the algebra $C$ is nontrivial only if $\operatorname{dim} q=3$ or 4 in which case the non trivial components are similar to

- $\left(\begin{array}{c}a b \\ k\end{array}\right)$ if $q$ is a neighbour of $\ll a, b \gg$.

- $\left(\begin{array}{c}a b \\ k(\sqrt{d})\end{array}\right)$ if $\operatorname{dim} q=4$ and $d=d_{ \pm} q \notin k^{* 2}$.

We then apply theorem 2.1 to get (i), (ii), and (iv).

We recall that $q$ is an Albert form if the dimension of $q$ is 6 and $d_{ \pm} q \in k^{* 2}$. In [Lag, Corollaire 6], Laghribi shows that if $q$ is an Albert form and if $L$ is the universal splitting field of $q$ over $k$ then one has an exact sequence

$$
k^{*} \stackrel{. U c(q)}{\longrightarrow} H^{3}(k, \mathbf{Z} / 2 \mathbf{Z}) \rightarrow H^{3}(L, \mathbf{Z} / 2 \mathbf{Z})
$$

where $c(q)$ is the clifford invariant of $q$. By [KR] this field coincides with the variety of Borel subgroups of $S O(q)$. We get the following result:

Proposition 7.8. Let $q$ be an Albert form, let $V$ be the variety of Borel subgroups in $S O(q)$ then

$$
\mathrm{CH}^{2}(V)_{\text {tors }}=\{0\}
$$




\section{AN EXPLICIT EXPRESSION IN A PARTICULAR CASE}

Definitions 8.1. For any field $K$ a field extension $L$ is a function field over $K$ if and only if it is generated by a finite number of elements as a field over $K$. If $L$ is a function field over $K$, then we denote by $\mathcal{P}(L / K)$ the set of discrete valuation rings of rank 1 such that

$$
K \subset A \subset L \text { and } \operatorname{Fr}(A)=L .
$$

If $A$ belongs to $\mathcal{P}(L / K)$ then $\kappa_{A}$ denotes the residue field of $A$ and if the characteristic of $K$ does not divide $n$

$$
\partial_{A}: H^{i}\left(L, \mu_{n}^{\otimes j}\right) \rightarrow H^{i-1}\left(\kappa_{A}, \mu_{n}^{\otimes j-1}\right)
$$

the residue map (see [CTO]). The unramified cohomology groups of $L$ over $K$ are then defined by

$$
H_{\mathrm{nr} / K}^{i}\left(L, \mu_{n}^{\otimes j}\right)=\bigcap_{A \in \mathcal{P}(L / K)} \operatorname{Ker}\left(\partial_{A}\right)
$$

In the rest of this paragraph we assume that the field $k$ is of characteristic different from 2, that the group $G$ is of the form $\mathrm{SL}_{1}(A) \times G^{\prime}$ where $A$ is a quaternion algebra $\left(\begin{array}{c}a b \\ k\end{array}\right)$ and that $V$ may be split in the product of a conic $C$ by a homogeneous variety $V^{\prime}$ so that the action of $G$ is the product of an action of $\mathrm{SL}_{1}(A)$ on $C$ and an action of $G^{\prime}$ on $V^{\prime}$. In this setting we shall now give a more explicit expression of the morphism from the homology of $(\mathcal{C})$ to $\mathrm{CH}^{2}(V)_{\text {tors }}$.

By [Ara, Satz 5.4] and Merkur'ev and Suslin [MS, theorem 12.1], one has an exact sequence

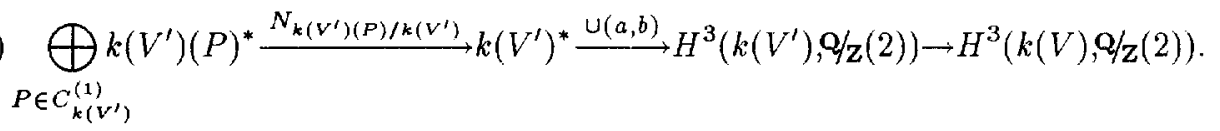

Let $\alpha$ be in the kernel of the canonical map from $H^{3}(k, \mathbf{Q} / \mathbf{Z}(2))$ to $H^{3}(k(V), \mathbf{Q} / \mathbf{Z}(2))$. Then $\alpha_{k\left(V^{\prime}\right)}$ may be written as $(a, b, f)$ for some $f$ in $k\left(V^{\prime}\right)$. Let $D$ be the divisor of $f$ on $V^{\prime}$. Since $\alpha$ comes from $H^{3}(k, \mathbf{Q} / \mathbf{Z}(2))$, for any point $P$ of codimension 1 in $V$ one has

$$
\partial_{P}(a, b, f)=0 \text {. }
$$

By [CTO, proposition 1.3], one gets that

$$
\forall P \in V^{\prime(1)},(a, b)_{k(P)} \neq 0 \Rightarrow \nu_{P}(f) \text { is even. }
$$

Let $p_{1}: V \rightarrow C$ and $p_{2}: V \rightarrow V^{\prime}$ be the natural projections. For any $P$ in $V^{\prime(1)}$, the conic $C_{k(P)}$ has a rational point if and only if $(a, b)_{k(P)}=0$. Thus we get that

$$
D \in \operatorname{Im}\left(p_{2^{*}}: \bigoplus_{P \in V^{(2)}} \mathbf{Z} \rightarrow \bigoplus_{P \in V^{\prime(1)}} \mathbf{Z}\right)
$$

Let $E$ belong to the inverse image of $D$ and $[E]$ be its class in $\mathrm{CH}^{2}(V) / p_{2}^{*}\left(\mathrm{CH}^{2}\left(V^{\prime}\right)\right)$.

Lemma 8.1. With the above notations, $[E]$ depends only on $\alpha$.

Proof. Let us first prove that for a fixed $f$ the class $[E]$ is independant of the choice of $E$. Let $E^{\prime}$ in $\bigoplus_{P \in V^{(2)}} \mathbf{Z}$ be such that $p_{2 *}\left(E^{\prime}\right)=p_{2_{*}}(E)$. We may then write

$$
E-E^{\prime}=\sum_{P \in V^{\prime(1)}} \sum_{\left\{Q \in V^{(2)} \mid p_{2 *}(Q) \in \mathbf{Z} P\right\}} n_{Q}^{P} Q+\sum_{P \in V^{\prime(2)}} n_{P} p_{2}^{*}(P)
$$


where for any $P$ in $V^{\prime(1)}, \sum_{\left\{Q \in V^{(2)} \mid p_{2 *}(Q) \in \mathbf{Z} P\right\}} n_{Q}^{P}[k(Q): k(P)]=0$. But for any $P \in V^{(1)}$, the Picard group of $C_{k(P)}$ is isomorphic to $\mathrm{Z}$ and thus there exists a function $f_{p}$ on $V_{P}$ such that

$$
\left(f_{P}\right)=\sum_{\left\{Q \in V^{(2)} \mid p_{2 *}(Q) \in \mathbf{Z} P\right\}} n_{Q}^{P} Q .
$$

Therefore the class of $E-E^{\prime}$ in $\mathrm{CH}^{2}(V) / p_{2}^{*}\left(\mathrm{CH}^{2}\left(V^{\prime}\right)\right)$ is trivial.

Let us now prove that $[E]$ is independant of the choice of $f$. Let $f^{\prime}$ be an element of $k\left(V^{\prime}\right)$ such that

$$
\alpha_{k\left(V^{\prime}\right)}=\left(a, b, f^{\prime}\right)
$$

By the exact sequence $(7)$, there exists a family $\left(f_{P}\right)_{P \in C_{k\left(V^{\prime}\right)}^{(1)}}$ in $\bigoplus_{P \in C_{k\left(V^{\prime}\right)}^{(1)}} k\left(V^{\prime}\right)(P)^{*}$ such that

$$
f / f^{\prime}=\prod_{P \in C_{k\left(V^{\prime}\right)}^{(1)}} N_{k\left(V^{\prime}\right)(P) / k\left(V^{\prime}\right)}\left(f_{P}\right)
$$

Then $E^{\prime}=E-\sum_{P \in C_{k\left(V^{\prime}\right)}^{(1)}} \operatorname{Div}\left(f_{P}\right)$ verifies $p_{2 *}\left(E^{\prime}\right)=\operatorname{Div}\left(f^{\prime}\right)$, but its class in $C^{2} H^{2}(V)$ is the same as the class of $E$.

We denote by

$$
\Phi: \operatorname{Ker}\left(H^{3}(k, \mathbf{Q} / \mathbf{Z}(2)) \rightarrow H^{3}(k(V), \mathbf{Q} / \mathbf{Z}(2))\right) \rightarrow \mathrm{CH}^{2}(V) / \mathrm{CH}^{2}\left(V^{\prime}\right)
$$

the induced map.

Theorem 8.2. With notation as above, the natural morphism from the homology of the complex $(\mathcal{C})$ to $\mathrm{CH}^{2}(V) / \mathrm{CH}^{2}\left(V^{\prime}\right)$ defined by theorem 2.1 coincides with the map induced by $\Phi$.

Proof. By [Kah, corollaire 3.2], there exists a canonical isomorphism

$$
H^{1}\left(\mathcal{G}, K_{2} k^{s}(V) / K_{2} k^{s}\left(V^{\prime}\right)\right) \stackrel{\sim}{\operatorname{Ker}}\left(H^{3}\left(k\left(V^{\prime}\right), \mathbf{Q} / \mathbf{Z}(2)\right) \rightarrow H^{3}(k(V), \mathbf{Q} / \mathbf{Z}(2))\right) .
$$

Moreover as in [Pe2, page 391] for any $g$ in $k^{s}\left(V^{\prime}\right)$ there is a commutative diagram

$$
\begin{array}{ccc}
H^{1}\left(\mathcal{G}, K_{1} k^{s}(V) / K_{1} k^{s}\left(V^{\prime}\right)\right) & \tilde{\rightarrow} & \operatorname{Ker}\left(\operatorname{Br}\left(k\left(V^{\prime}\right)\right) \rightarrow \operatorname{Br}(k(V))\right) \\
\downarrow \cup g & \downarrow \cup g & \\
H^{1}\left(\mathcal{G}, K_{2} k^{s}(V) / K_{2} k^{s}\left(V^{\prime}\right)\right) & \sim & \operatorname{Ker}\left(H^{3}\left(k\left(V^{\prime}\right), \mathbf{Q} / \mathbf{Z}(2)\right) \rightarrow H^{3}(k(V), \mathbf{Q} / \mathbf{Z}(2))\right) .
\end{array}
$$

But there is a surjection

$$
\left(\operatorname{Pic} C^{s}\right)^{\mathcal{G}} \rightarrow H^{1}\left(\mathcal{G}, K_{1} k^{s}(V) / K_{1} k^{s}\left(V^{\prime}\right)\right) .
$$

Moreover Pic $C^{s}$ is a free $\mathrm{Z}$-module of rank 1 with a trivial $\mathcal{G}$ action and the image of one of its generator in $\operatorname{Br}\left(k\left(V^{\prime}\right)\right)$ is $(a, b)$.

We use again the symbols $\alpha, f$ and $E$ introduced before lemma 8.1 . Let $\beta$ be the image of one generator of $\operatorname{Pic} C^{s}$ in $H^{1}\left(\mathcal{G}, K_{1} k^{s}(V) / K_{1} k^{s}\left(V^{\prime}\right)\right)$. Let $\gamma$ be the image of $\alpha$ in $H^{1}\left(\mathcal{G}, K_{2} k^{s}(V) / K_{2} k^{s}\right)$. Then the image of $\gamma$ in $H^{1}\left(\mathcal{G}, K_{2} k^{s}(V) / K_{2} k^{s}\left(V^{\prime}\right)\right)$ is $\beta \cup f$.

The conic $C$ may be defined by the homogeneous equation

$$
X^{2}-a Y^{2}-b Z^{2}=0 .
$$

Let $\sqrt{a}$ be a square root of $a$ in $k^{s}$. Then the cocycle

$$
\begin{aligned}
\tilde{\beta}: \mathcal{G} & \rightarrow k^{s}(V)^{*} / k^{s}\left(V^{\prime}\right)^{*} \\
\sigma & \mapsto \begin{cases}{[1]} & \text { if } \sigma(\sqrt{a})=\sqrt{a} \\
{\left[\frac{x-\sqrt{a} Y}{2}\right]} & \text { otherwise. }\end{cases}
\end{aligned}
$$


represents $\beta$. The element $\beta \cup f$ is thus given by the cocycle

$$
\begin{aligned}
\widetilde{\beta \cup f}: \mathcal{G} & \rightarrow K_{2} k^{s}(V) / K_{2} k^{s}\left(V^{\prime}\right) \\
\sigma & \mapsto \begin{cases}{[0]} & \text { if } \sigma(\sqrt{a})=\sqrt{a}, \\
{\left[\left\{\frac{x-\sqrt{a} Y}{Z}, f\right\}\right]} & \text { otherwise. }\end{cases}
\end{aligned}
$$

But the map

$$
\Psi: H^{1}\left(\mathcal{G}, K_{2}\left(k^{s}(V)\right) / K_{2} k^{s}\right) \rightarrow \mathrm{CH}^{2}(V)
$$

is defined as follows (see [CTR, page 188]). One considers the kernel $\mathcal{Z}$ and the image $\mathcal{I}$ of the morphism

$$
\bigoplus_{x \in V^{(1)}} k(x)^{*} \rightarrow \bigoplus_{x \in V^{(2)}} \mathbf{Z}
$$

given by Gersten-Quillen spectral sequence, as well as the corresponding groups $\mathcal{Z}_{k}$ s and $\mathcal{I}_{k^{s}}$ over $k^{s}$. We have a short exact sequence

$$
0 \rightarrow K_{2} k^{s}(V) / K_{2} k^{s} \rightarrow \mathcal{Z}_{k^{s}} \rightarrow H^{1}\left(V, \mathcal{K}_{2}\right) \rightarrow 0 .
$$

Then the isomorphism

$$
\Psi: H^{1}\left(\mathcal{G}, \mathcal{Z}_{k^{s}}\right) \stackrel{\sim}{\rightarrow} \operatorname{Ker}\left(\mathrm{CH}^{2}(V) \rightarrow \mathrm{CH}^{2}\left(V_{k^{s}}\right)\right)
$$

is yielded by diagram chases in the following two commutative diagrams which have exact lines

$$
\begin{aligned}
& 0 \rightarrow \mathcal{Z}_{k^{s}}^{\mathcal{G}} \rightarrow\left(\bigoplus_{x \in V_{k^{s}}^{(1)}} k^{s}(x)^{*}\right)^{\mathcal{G}} \rightarrow \mathcal{I}_{k^{s}}^{\mathcal{G}} \stackrel{\partial}{\rightarrow} H^{1}\left(\mathcal{G}, \mathcal{Z}_{k^{s}}\right) \rightarrow 0 \\
& 0 \rightarrow \mathcal{Z} \rightarrow \bigoplus_{x \in V^{(1)}} k(x)^{*} \rightarrow \uparrow_{\mathcal{I}} \rightarrow 0
\end{aligned}
$$

and

$$
\begin{aligned}
& 0 \rightarrow \mathcal{I}_{k^{s}}^{\mathcal{G}} \rightarrow\left(\bigoplus_{x \in V_{k^{s}}^{(2)}} \mathbf{Z}\right)^{\mathcal{G}} \rightarrow \mathrm{CH}^{2}\left(V_{k^{s}}\right) \\
& 0 \rightarrow \uparrow_{\mathcal{I}} \bigoplus_{x \in V^{(2)}} \mathbf{Z} \rightarrow \mathrm{CH}^{2}(V) \rightarrow 0 .
\end{aligned}
$$

We put $\mathcal{C}(1)=\bigoplus_{x \in V^{(1)}} k(x)^{*}$ and $\mathcal{C}(2)=\bigoplus_{x \in V^{(2)}} \mathbf{Z}$ and take a similar notation for the corresponding groups over $k^{s}$. Let $\mathcal{C}^{\prime}(1), \mathcal{C}_{k^{s}}^{\prime}(1), \mathcal{C}^{\prime}(2), \mathcal{C}_{k}^{\prime} s(2), \mathcal{Z}^{\prime}, \mathcal{Z}_{k^{s}}^{\prime}, \mathcal{I}^{\prime}$ and $\mathcal{I}_{k^{*}}^{\prime}$ be the corresponding objects for $V^{\prime}$. Since the map from $\mathcal{I}^{\prime}$ to $\mathcal{I}$ is injective, we have exact sequences

$$
0 \rightarrow \mathcal{Z} / \mathcal{Z}^{\prime} \rightarrow \mathcal{C}(1) / \mathcal{C}^{\prime}(1) \rightarrow \mathcal{I} / \mathcal{I}^{\prime} \rightarrow 0
$$

and

$$
\mathcal{I} / \mathcal{I}^{\prime} \rightarrow \mathcal{C}(2) / \mathcal{C}^{\prime}(2) \rightarrow \mathrm{CH}^{2}(V) / \mathrm{CH}^{2}\left(V^{\prime}\right) \rightarrow 0
$$

as well as the corresponding ones over $k^{s}$. Moreover Hilbert's theorem 90 implies that

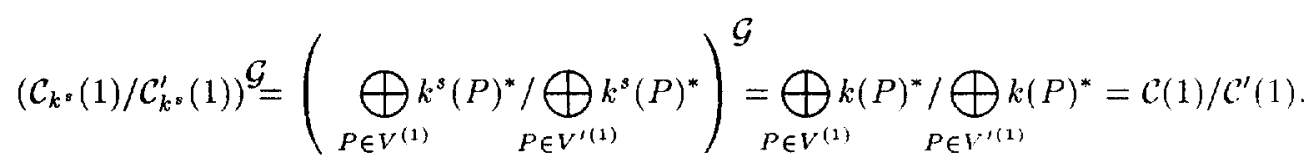


Thus we get the following two commutative diagrams of complexes the first of which has exact lines:

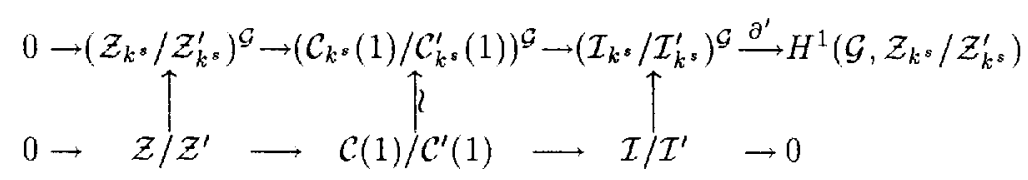

and

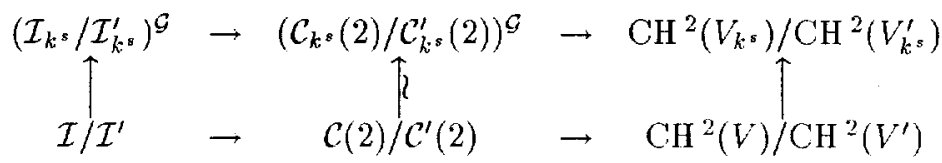

which defines a map from $\operatorname{Im} \partial^{\prime}$ to $\mathrm{CH}^{2}(V) / \mathrm{CH}^{2}\left(V^{\prime}\right)$. Moreover there are obvious morphisms of diagrams from (8) to (10) and from (9) to (11). Thus we get a commutative diagram

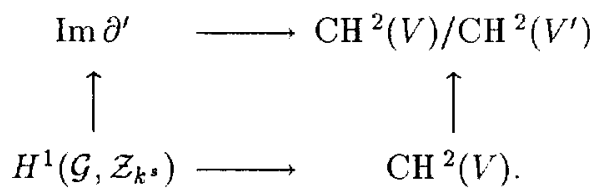

It remains to show that the image of $\beta \cup f$ in $\mathrm{CH}^{2}(V) / \mathrm{CH}^{2}\left(V^{\prime}\right)$ is given by the class of $E$. The image of $\beta \cup f$ in $H^{1}\left(\mathcal{G}, \mathcal{Z}_{k^{s}} / \mathcal{Z}_{k^{s}}^{\prime}\right)$ is given by the cocycle

$$
\widetilde{\beta \cup f^{\prime}}: \sigma \mapsto \begin{cases}0 & \text { if } \sigma(\sqrt{a})=\sqrt{a}, \\ {\left[\sum_{P \in V_{k^{\prime}}^{(1)}} \lambda_{P}\right]} & \text { otherwise. }\end{cases}
$$

where

$$
\lambda_{P}=\left\{\begin{array}{l}
\left(\frac{Z}{X-\sqrt{a} Y}\right)^{\nu_{Q}(f)} \text { if } P=p_{2}^{-1}(Q), \\
f \text { if } P=p_{1}^{-1}((\sqrt{a}: 1: 0)), \\
f^{-1} \text { if } P=p_{1}^{-1}((\sqrt{a}:-1: 0)), \\
1 \text { otherwise. }
\end{array}\right.
$$

We may write $E$ in the form $E_{0}+\sum_{Q \in V^{\prime}(1)} E_{Q}$ where $p_{2 *}\left(E_{0}\right)=0$ and for any $Q$ in $V^{(1)}$, the support of $E_{Q}$ is included in $p_{2}^{-1}(Q)$ and $p_{2 *}\left(E_{Q}\right)$ is equal to $\nu_{Q}(f) Q$. Thus for any $Q$ in $V^{(1)}$ there exists a function $g_{Q}$ in $k^{s}(Q)(C)$ such the divisor of $g_{Q}$ over $k^{s}(Q)$ is

$$
-\nu_{Q}(f)(\sqrt{a}:-1: 0)+E_{Q}
$$

and they may be choosen to be trivial except for a finite number. Then one considers the element $\gamma$ of $\mathcal{C}_{k} s(1) / \mathcal{C}_{k}^{\prime} s(1)$ defined by $\gamma=\left[\sum_{P \in V(1)} \gamma_{P}\right]$ where

$$
\gamma_{P}=\left\{\begin{array}{l}
f \text { if } P=p_{1}^{-1}((\sqrt{a}:-1: 0)), \\
g_{Q} \text { if } P=p_{2}^{-1}(Q), \\
1 \text { otherwise. }
\end{array}\right.
$$

Then for any $\sigma$ in $\mathcal{G}$ such that $\sigma(\sqrt{a})=-\sqrt{a}$ and any $Q$ in $V^{\prime(1)}$ the divisor of ${ }^{\sigma} g_{Q} g_{Q}^{-1}$ over $k^{s}(Q)$ coincides with the one of $\left(\frac{X-\sqrt{a} Y}{Z}\right)^{-\nu_{Q}(f)}$. Thus, for any $\sigma \in \mathcal{G}$,

$$
\widetilde{\beta \cup f}^{\prime}(\sigma)={ }^{\sigma} \gamma \gamma^{-1} \text {. }
$$


Moreover the image of $\gamma$ in $\mathcal{I}_{k^{s}} / \mathcal{I}_{k^{s}}^{\prime}$ is invariant under $\mathcal{G}$ and comes from $E$ by the natural map

$$
\bigoplus_{P \in V^{(2)}} \mathbf{Z} \rightarrow\left(\mathcal{C}_{k^{s}}(1) / \mathcal{C}_{k^{s}}^{\prime}(1)\right)^{\mathcal{G}}
$$

which implies the result.

\section{Application to NEGLigible Classes}

We shall now apply the results of the preceding sections to the study of negligible classes in the cohomology of a central extension of an $\mathbf{F}_{p}$ vector space by another.

9.1. Products of generic Severi-Brauer varieties. Let $p$ be a prime number and $k$ be a field of characteristic different from $p$. If $p=2$ we assume that the field contains a primitive fourth root of one and in general that it contains a primitive $p$-th root of one $\xi$. Let $m$ be an integer, $X_{1}, \ldots, X_{n}$ be indeterminates and $K$ be the field $k\left(X_{1}, \ldots, X_{n}\right)$. We fix an integer $m$ and monomials $A_{i}, B_{i}$ for $1 \leqslant i \leqslant m$. We then consider the cyclic simple algebras $D_{i}=A_{\xi}\left(A_{i}, B_{i}\right)$ generated by two elements $I$ and $J$ with the relations

$$
I^{p}=A_{i}, J^{p}=B_{i} \text { and } I J=\xi J I,
$$

we denote by $V_{i}$ the corresponding Severi-Brauer variety and by $V$ the product of these varieties. Using Amitsur's theorem [Am, theorem 9.3] one gets (see [Pe1, lemma 8]) that

$$
\operatorname{Ker}(\operatorname{Br} K \rightarrow \operatorname{Br} K(V))=<\left(A_{i}, B_{i}\right), 1 \leqslant i \leqslant m>
$$

and the complex $\mathcal{C}$ may be written as

$$
<\left(A_{i}, B_{i}\right), 1 \leqslant i \leqslant m>\otimes K^{*} \stackrel{\cup}{\rightarrow} H^{3}(K, \mathbf{Q} / \mathbf{Z}(2)) \rightarrow H^{3}(K(V), \mathbf{Q} / \mathbf{Z}(2))
$$

9.2. Connection with negligible classes. Let $U$ be an $\mathbf{F}_{p}$ vector space with a basis $u_{1}, \ldots, u_{n}$. Let $\Phi_{K}^{1}$ be the morphism from the dual $U^{\vee}$ of $U$ to $K^{*} / K^{* 2}$ which sends $u_{i}^{\vee}$ of the dual basis to $\left(X_{i}\right)$. Let

$$
\Phi_{K}^{i}:\left(\Lambda^{i} U\right)^{\vee} \rightarrow H^{i}\left(K, \mu_{p}\right)
$$

be the induced map (see [Pe1, page 250]). This is an injection by [Pe1, lemma 7]. Moreover

$$
\operatorname{Ker}(\operatorname{Br}(K) \rightarrow \operatorname{Br}(K(V))) \subset \operatorname{Im} \Phi_{K}^{2}
$$

Let $V$ be the dual of the inverse image of this kernel by $\Phi_{K}^{2}$. We then have an injection

$$
V^{\vee} \hookrightarrow\left(\Lambda^{2} U\right)^{\vee}
$$

and a surjective map

$$
\Lambda^{2} U \rightarrow V
$$

which gives an element $\gamma$ in $\Lambda^{2}\left(U^{\vee}\right) \otimes V$.

For any vector space $W$ over a field $F$ let $A C^{*}(W)$ be the quotient of the tensor algebra $T^{*}(W)$ by the ideal generated by $x \otimes y-y \otimes x$ for all $x, y$ in $W$. Then there is a surjective morphism

$$
A C^{*}(W) \rightarrow \Lambda^{*}(W)
$$

which is an isomorphism if the characteristic of $F$ is not 2 and a natural map

$$
A C^{*}\left(W^{\vee}\right) \rightarrow H^{*}(W, F) \text {. }
$$

Let $\tilde{\gamma}$ be a lift of $\gamma$ in $A C^{*}\left(U^{\vee}\right) \otimes V$. We also denote by $\tilde{\gamma}$ its image in $H^{2}(U, V)$. There is a natural surjection

$$
H^{2}(U, V) \rightarrow \Lambda^{2}\left(U^{\vee}\right) \otimes V
$$


(see [Bro, exercise IV.3.8]). The image of $\tilde{\gamma}$ by this map coincides with $\gamma$. Let

$$
0 \rightarrow V \stackrel{3}{\rightarrow} G \stackrel{\pi}{\rightarrow} U \rightarrow 0
$$

be the central extension corresponding to $\tilde{\gamma}$.

Definition 9.1. If $H$ is a finite group, $M$ a $H$-module and $E$ a field, a class $\lambda$ in $H^{i}(H, M)$ is said to be E-negligible if and only if for any extension $F$ of $E$ and any morphism

$$
\rho: \operatorname{Gal}\left(F^{s} / F\right) \rightarrow H
$$

the image of $\lambda$ by $\rho^{*}$ is zero in $H^{i}(F, M)$. by

If $E$ is a field over $k$ and $\boldsymbol{a}=\left(a_{i}\right)_{1 \leqslant i \leqslant n}$ a family of inversible elements of $E$. we denote

$$
\bigoplus_{i \geqslant 0} \Phi_{E, a}^{i}: \bigoplus_{i \geqslant 0} \Lambda^{i} U^{\vee} \rightarrow \bigoplus_{i \geqslant 0} H^{i}\left(E, \mu_{p}^{\otimes i}\right)
$$

the morphism of graded algebras which sends $u_{j}^{\vee}$ onto $a_{j}$.

Proposition 9.1. Let $\tilde{\lambda}$ belong to $A C^{i} U^{\vee}$ and $\lambda$ be its image in $\Lambda^{i} U^{\vee}$. The following three assertions are equivalent:

(1) The image of $\tilde{\lambda}$ in $H^{i}\left(G, \mu_{p}^{\otimes i}\right)$ is k-negligible.

(2) For any field $E$ over $k$ and any family $a=\left(a_{i}\right)_{1 \leqslant i \leqslant n}$ of inversible elements in E such that

$$
\Phi_{E, a}^{2}\left(V^{\vee}\right)=0
$$

one has

$$
\Phi_{E, a}^{i}(\lambda)=0
$$

(3) One has $\Phi_{K(V), X}^{i}(\lambda)=0$.

Proof. - Let us first prove the equivalence of the first two assertions. Let $E$ be an extension of $k$. There is a natural correspondance between the morphisms $\rho_{1}$ from $\operatorname{Gal}\left(F^{s} / F\right)$ to $U$ and the families $\left(a_{i}\right)_{1 \leqslant i \leqslant n}$ of elements of $E^{*} / E^{* p}$. Let us first prove the following lemma:

Lemma 9.2. With the notation as above, the morphism

$$
\rho_{1}: \operatorname{Gal}\left(F^{s} / F\right) \rightarrow U
$$

may be lifted in a morphism

$$
\rho: \operatorname{Gal}\left(F^{s} / F\right) \rightarrow G
$$

if and only if

$$
\Phi_{E, a}^{2}\left(V^{\vee}\right)=0 .
$$

Proof. The morphism $\rho_{1}$ may be lifted to $G$ if and only if the image of $\tilde{\gamma}$ in $H^{2}(F, V)$ is trivial. Let us choose a basis $\left(v_{1}, \ldots, v_{m}\right)$ of $V$ and let $\left(v_{1}^{v}, \ldots, v_{m}^{v}\right)$ be the dual basis. The condition is equivalent to the triviality of $v_{i *}^{\vee}\left(\rho_{1}^{*}(\tilde{\gamma})\right)$ for $1 \leqslant i \leqslant m$ that is the triviality of $\rho_{1}^{*}\left(v_{i *}^{\vee}(\tilde{\gamma})\right)$ for $1 \leqslant i \leqslant m$ which is equivalent to $\Phi_{E, a}^{2}\left(v_{i}^{\vee}\right)=0$. 
End of the proof of proposition 9.1. Let us assume that the first assertion is true and let $E$ be a field over $k$ and $\boldsymbol{a}$ a family of inversible elements such that the hypothesis of the second assertion are verified. By the preceding lemma, the morphism $\operatorname{Gal}\left(E^{s} / E\right) \rightarrow U$ defined by $a$ may be lifted to $G$. But by the first assertion the image of $\tilde{\lambda}$ in $H^{i}(G, \mathbf{Z} / p \mathbf{Z})$ is sent to 0 in $H^{i}\left(E, \mu_{p}^{\otimes i}\right)$.

Let us assume that the second assertion is true, let $E$ be a field extension of $k$ and $\rho: \mathrm{Gal}\left(E^{s} / E\right) \rightarrow G$ be a morphism. The map $\rho$ induces a morphism

$$
\operatorname{Gal}\left(E^{s} / E\right) \rightarrow U
$$

corresponding to a family $\boldsymbol{a}$. By the lemma $\Phi_{E, \boldsymbol{a}}^{2}\left(V^{\vee}\right)=0$. Thus $\Phi_{E, \boldsymbol{a}}^{i}(\lambda)=0$ and the image of $\tilde{\lambda}$ in $H^{i}\left(E, \mu_{p}^{\otimes i}\right)$ is trivial.

- It is clear that the second assertion implies the third. Let us prove the opposite implication. First the fact that $\Phi_{K(V), X}^{i}(\lambda)=0$ implies that $\Phi_{E \otimes_{k} K(V), X}^{i}(\lambda)=0$. Thus we are reduced to the case where $k=E$.

Let $A_{1} \in \mathcal{P}(K / k)$ be the discrete valuation ring of rank one defined by the divisor $X_{1}=a_{1}$. Consider the algebras $\mathcal{A}_{\xi}^{1}\left(A_{i}, B_{i}\right)$ defined over $A_{1}$ by the generators $I$ and $J$ and the relations

$$
I^{p}=A_{i}, J^{p}=B_{i} \text { and } I J=\xi J I .
$$

It defines a Severi-Brauer scheme $\mathcal{V}_{i}^{\prime}$ over $\operatorname{Spec} A_{1}$. Let $\mathcal{V}^{1}$ be the product of these schemes. Then the special fiber of $\mathcal{V}^{1}$ defines a local ring $B_{1}$ in $\mathcal{P}(K(V) / k)$ over $A_{1}$ such that $B_{1}$ is unramified over $A_{1}$ and the residue field $\kappa_{B}$ is the function field over $k\left(X_{2}, \ldots, X_{n}\right)$ of the product $V^{1}$ of the Severi-Brauer varieties defined by the algebras $A_{\xi}\left(A_{i}\left(a_{1}, X_{2}, \ldots, X_{n}\right), B_{i}\left(a_{1}, X_{2}, \ldots, X_{n}\right)\right)$. In a similar way we construct discrete valuation rings of rank one $A_{i}$ in $\mathcal{P}\left(\kappa_{A_{i-1}} / k\right)$ and $B_{i} \in \mathcal{P}\left(\kappa_{B_{i-1}} / k\right)$ so that $\kappa_{A_{i}}=$ $k\left(X_{i+1}, \ldots, X_{n}\right)$ and $\kappa_{B_{i}}=\kappa_{A_{i}}\left(V^{i}\right)$ where $V^{i}$ is defined as $V^{1}$. We put $\kappa_{A_{0}}=K$ and $\kappa_{B_{0}}=K(V)$. Let us assume that

$$
\Phi_{r_{B_{i}},\left(a_{1}, \ldots, a_{i}, X_{i+1}, \ldots, X_{n}\right)}^{i}(\lambda)=0 .
$$

Then taking the completion of $\kappa_{B_{1}}$ for $B_{i+1}$

$$
\Phi_{\hat{\kappa}_{B_{i}},\left(a_{1}, \ldots, a_{i}, X_{i+1}, \ldots, X_{n}\right)}^{i}(\lambda)=0 .
$$

But the field $\hat{\kappa}_{B_{i}}$ is isomorphic to $\kappa_{B_{i+1}}\left(\left(X_{i+1}-a_{i+1}\right)\right)$. I hus the natural surjection

$$
\operatorname{Gal}\left(\hat{\kappa}_{B_{i}}^{s} / \hat{\kappa}_{B_{i}}\right) \rightarrow \operatorname{Gal}\left(\kappa_{B_{i+1}}^{s} / \kappa_{B_{i+1}}\right)
$$

has a section and the map

$$
H^{i}\left(\kappa_{B_{i+1}}, \mu_{p}^{\otimes i}\right) \rightarrow H^{i}\left(\hat{\kappa}_{B_{i}}, \mu_{p}^{\otimes i}\right)
$$

is an injection. Thus

$$
\Phi_{\kappa_{B_{i+1}}^{i},\left(a_{1}, \ldots, a_{i}, a_{i+1}, X_{2+2}, \ldots, X_{n}\right)}^{i}(\lambda)=0 .
$$

Therefore by induction, we get that $\Phi_{\kappa_{B_{n}}}^{i}, a(\lambda)=0$. But, since $\Phi_{k, \mathbf{a}}^{2}(V)=0, k_{B_{n}}$ is rational on $k$ and $\Phi_{k, a}^{i}(\lambda)=0$.

Lemma 9.3. The images in $\Lambda^{3}\left(U^{\vee}\right)$ of the kernels of the natural maps

$$
H^{3}(U, \mathbf{Z} / p \mathbf{Z}) \rightarrow H^{3}(G, \mathbf{Z} / p \mathbf{Z})
$$

and

are equal to $U^{\vee} \wedge V^{\vee}$.

$$
H^{3}(U, \mathbf{Z} / p \mathbf{Z}) \rightarrow H^{3}(G, \mathbf{Q} / \mathbf{Z})
$$


Proof. The element $\tilde{\gamma}$ in $A C^{2}\left(U^{\vee}\right) \Theta V$ defines a morphism

$$
\tilde{\gamma}: V^{\vee} \rightarrow A C^{2}\left(U^{\vee}\right)
$$

which is injective. We then consider the Hochschild-Serre spectral sequences

$$
E_{\mathbf{2}}^{p, q}(\mathbf{Z} / p \mathbf{Z})=H^{p}\left(U, H^{q}(V, \mathbf{Z} / p \mathbf{Z})\right) \Rightarrow H^{p+q}(G, \mathbf{Z} / p \mathbf{Z})
$$

and

$$
E_{2}^{p, q}(\mathbf{Q} / \mathbf{Z})=H^{p}\left(U, H^{q}(V, \mathbf{Q} / \mathbf{Z})\right) \Rightarrow H^{p+q}(G, \mathbf{Q} / \mathbf{Z})
$$

Moreover the natural map $\mathbf{Z} / p \mathbf{Z} \rightarrow \mathbf{Q} / \mathbf{Z}$ induces a morphism of spectral sequences from the first one to the second one. If $p=2$ there is a natural isomorphism

$$
S^{p}\left(U^{\vee}\right) \otimes S^{q}\left(V^{\vee}\right) \stackrel{\sim}{\rightarrow} H^{p}\left(U, H^{q}(V, \mathbf{Z} / 2 \mathbf{Z})\right)
$$

(see [Car2, théorème 2]) and if $p \neq 2$ isomorphisms

$$
U^{\vee} \oplus \Lambda^{2}\left(U^{\vee}\right) \stackrel{\delta \oplus U}{\longrightarrow} H^{2}(U, \mathbf{Z} / p \mathbf{Z})
$$

where $\delta$ is the Bockstein operator and by [Car1, théorème 2],

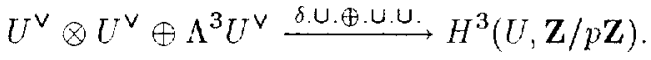

- By [Bro, page 60], one has an exact sequence

$$
0 \rightarrow \operatorname{Ext}_{\mathbf{Z}}^{1}\left(H_{n-1}(U, \mathbf{Z}), \mathbf{Q} / \mathbf{Z}\right) \rightarrow H^{n}(U, \mathbf{Q} / \mathbf{Z}) \rightarrow \operatorname{Hom}\left(H_{n}(U, \mathbf{Z}), \mathbf{Q} / \mathbf{Z}\right) \rightarrow 0 .
$$

But $\operatorname{Ext}_{\mathbf{Z}}^{1}\left(H_{n-1}(U, \mathbf{Z}), \mathbf{Q} / \mathbf{Z}\right)$ is trivial and by [Bro, pages 122 and 123 ], $\Lambda^{i} U \sim H_{i}(U, \mathbf{Z})$ if $i=1$ or 2 and $\Lambda^{i} U \hookrightarrow H_{i}(U, \mathbf{Z})$ for $i \geqslant 3$. We get isomorphisms

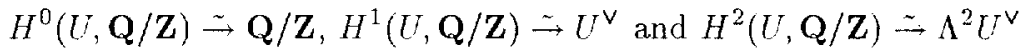

as well as surjections

$$
H^{i}(U, \mathbf{Q} / \mathbf{Z}) \rightarrow \Lambda^{i} U^{\vee}
$$

Similarly, one gets

$$
H^{1}\left(U, H^{1}(V, \mathbf{Q} / \mathbf{Z})\right) \stackrel{\sim}{\rightarrow} U^{\vee} \otimes V^{\vee} \text { and } H^{2}(V, \mathbf{Q} / \mathbf{Z}) \tilde{\rightarrow} \Lambda^{2} V^{\vee}
$$

- We shall now prove that the map

$$
V^{\vee} \rightarrow A C^{2}\left(U^{\vee}\right)
$$

induced by the first spectral sequence is the one defined by $-\tilde{\gamma}$. We use the same filtration on $\bigoplus_{n \in N} C^{n}(G, \mathbf{Z} / p \mathbf{Z})$ as in section 4 . Let us write $\tilde{\gamma}=\sum_{i=1}^{t} f_{i} \cdot g_{i} \otimes v_{i}$ with $f_{i}, g_{i}$ in $U^{\vee}$ and $v_{i}$ in $V$. Let $s: U \rightarrow G$ be a set-theoritic section of $\pi$ such that $s(0)=\epsilon$ and

$$
\forall u, u^{\prime} \in U, s(u) s\left(u^{\prime}\right)=j\left(\sum_{i=1}^{t} f_{i}(u) g_{i}(u) v_{i}\right) s\left(u u^{\prime}\right) .
$$

Let $h$ belong to $V^{\vee}=H^{0}\left(U, H^{1}(V, \mathbf{Z} / p \mathbf{Z})\right) \sim E_{2}^{0,1}$. For any $g$ in $G$ let $\tau(g) \in V$ denote $g s(\pi(g))^{-1}$. Then $h$ is represented in $C^{1}(G, \mathbf{Z} / p \mathbf{Z})$ by the cocycle $\tilde{h}$ defined by

$$
\begin{aligned}
G & \rightarrow \mathbf{Z} / p \mathbf{Z} \\
g & \mapsto h(\tau(g)) .
\end{aligned}
$$


But $\tau\left(g g^{\prime}\right)=\tau(g)+\tau\left(g^{\prime}\right)+\sum_{i=1}^{t} f_{i}(\pi(g)) g_{i}\left(\pi\left(g^{\prime}\right)\right) v_{i}$. Thus

$$
\begin{aligned}
d \tilde{h}\left(g, g^{\prime}\right) & =h\left(\tau\left(g^{\prime}\right)\right)-h\left(\tau\left(g g^{\prime}\right)\right)+h(\tau(g)) \\
& =-h\left(\sum_{i=1}^{t} f_{i}(\pi(g)) g_{i}\left(\pi\left(g^{\prime}\right)\right) v_{i}\right)
\end{aligned}
$$

which is sent in $H^{2}(U, \mathbf{Z} / p \mathbf{Z})$ on the image of the element of $A C^{2}\left(U^{\vee}\right)$ given by

$$
-\sum_{i=1}^{t} h\left(v_{i}\right) f_{i} \cdot g_{i}
$$

But it is the opposite of the image of $h$ by $\dot{\gamma}$.

- Since the Hochschild-Serre spectral sequence is compatible with the cup-product, the map

$$
H^{1}\left(U, H^{1}(V, \mathbf{Z} / p \mathbf{Z})\right) \rightarrow H^{3}(U, \mathbf{Z} / p \mathbf{Z})
$$

is induced by the map

$$
\begin{aligned}
U^{\vee} \otimes V^{\vee} & \rightarrow A C^{3} U^{\vee} \\
u \otimes v & \mapsto-u \cdot \tilde{\gamma}(v) .
\end{aligned}
$$

- Also thanks to this compatibility, the composite morphism

$$
A C^{2}\left(V^{\vee}\right) \rightarrow H^{0}\left(U, H^{2}(V, \mathbf{Z} / p \mathbf{Z})\right) \rightarrow H^{2}\left(U, H^{1}(V, \mathbf{Z} / 2 \mathbf{Z})\right)
$$

is given by the opposite of the map

$$
\begin{aligned}
A C^{2}\left(V^{\vee}\right) & \rightarrow A C^{2}\left(U^{\vee}\right) \otimes V^{\vee} \\
x y & \mapsto \tilde{\gamma}(x) \otimes y-\tilde{\gamma}(y) \otimes x .
\end{aligned}
$$

- By the expression of the map

$$
H^{1}\left(U, H^{1}(V, \mathbf{Z} / p \mathbf{Z})\right) \rightarrow H^{3}(U, \mathbf{Z} / p \mathbf{Z})
$$

we get that $U^{\vee} \wedge V^{\vee}$ is contained in the image in $\Lambda^{3} U^{\vee}$ of the kernel of the inflation map

$$
H^{3}(U, \mathbf{Z} / p \mathbf{Z}) \rightarrow H^{3}(G, \mathbf{Z} / p \mathbf{Z})
$$

Thus it suffices to prove that the image in $\Lambda^{3} U^{\vee}$ of

$$
\operatorname{Ker}\left(H^{3}(U, \mathbf{Z} / p \mathbf{Z}) \rightarrow H^{3}(G, \mathbf{Q} / \mathbf{Z})\right)
$$

is contained in $U^{\vee} \wedge V^{\vee}$. But, by (12) and (13), we already know that the image of the composite map

$$
H^{1}\left(U, H^{1}(V, \mathbf{Q} / \mathbf{Z})\right) \rightarrow H^{3}(U, \mathbf{Q} / \mathbf{Z}) \rightarrow \Lambda^{3} U^{\vee}
$$

is contained in $U^{\vee} \wedge V^{\vee}$. Also the computation of the map

$$
A C^{2}\left(V^{\vee}\right) \rightarrow H^{2}\left(U, H^{1}(V, \mathbf{Z} / p \mathbf{Z})\right)
$$

shows that the map

$$
H^{2}(V, \mathbf{Q} / \mathbf{Z}) \rightarrow H^{2}\left(U, H^{1}(V, \mathbf{Q} / \mathbf{Z})\right)
$$

is induced by the map

$$
\begin{aligned}
\Lambda^{2} V^{\vee} & \rightarrow A C^{2}\left(U^{\vee}\right) \otimes V^{\vee} \\
x y & \mapsto \tilde{\gamma}(x) \otimes y-\tilde{\gamma}(y) \otimes x
\end{aligned}
$$

which is injective, since $\tilde{\gamma}$ is injective. Therefore $E_{3}^{0,2}(\mathbf{Q} / \mathbf{Z})$ is trivial and the result is proved. 
Notation. Let $\left.\left(V^{\vee} \wedge U^{\vee}\right)\right)_{\operatorname{dec}}^{\perp} \subset \Lambda^{3} U$ be the subgroup of $\left(V^{\vee} \wedge U^{\vee}\right)^{\perp}$ generated by the elements of the form $u \wedge v$, for $u \in \Lambda^{2} U, v \in U$. Let $K_{\max }^{3}$ be its orthogonal in $\Lambda^{3} U^{\vee}$.

Proposition 9.4. The inverse image in $\Lambda^{3} U^{\vee}$ of $H_{\mathrm{nr} / k}^{3}\left(K(V), \mu_{p}^{\otimes 3}\right)$ is equal to $K_{\max }^{3}$. In particular one has

$$
V^{\vee} \wedge U^{\vee} \subset \operatorname{Ker} \Phi_{K(V)}^{3} \subset K_{\max }^{3}
$$

Proof. The inclusion $V^{\vee} \wedge U^{\vee} \subset \operatorname{Ker} \Phi_{K(V)}^{3}$ is clear. Therefore

$$
\left(\operatorname{Ker} \Phi_{K(V)}^{3}\right)^{\perp} \subset\left(V^{\vee} \wedge U^{\vee}\right)^{\perp}
$$

and

$$
\left(\operatorname{Ker} \Phi_{K(V)}^{3}\right)_{\mathrm{dec}}^{\perp} \subset\left(V^{\vee} \wedge U^{\vee}\right)_{\mathrm{dec}}^{\perp}
$$

Thus for any $f$ in $K_{\text {max }}^{3}$, the restriction of $f$ to $\left(K e r \Phi_{K(V)}^{3}\right)_{\text {dec }}^{\perp}$ is trivial and by [Pe1, theorem 2 and remark p. 251] the image of $f$ in $H^{3}\left(K(V), \mu_{p}^{\otimes 3}\right)$ is unramified over $k$.

Let $\lambda \notin K_{\text {max }}^{-3}$. We want to show that its image is ramified. There exist $p \in \Lambda^{2} U$ and $u_{1}^{\prime} \in U$ such that $\left\langle\lambda, p \wedge u_{1}^{\prime}>\neq 0\right.$ and $p \wedge u_{1}^{\prime}$ belongs to $\left(V^{\vee} \wedge U^{\vee}\right)^{\perp}$. We complete $\left(u_{1}^{\prime}\right)$ in a basis $\left(u_{1}^{\prime}, \ldots, u_{n}^{\prime}\right)$ of $U$. We may choose these elements so that they can be lifted in a basis of $Z^{n}$. Let $M_{1}, \ldots, M_{n}$ be the monomials in $X_{1}, \ldots, X_{n}, X_{1}^{-1}, \ldots, X_{n}^{-1}$ given by this basis of $Z^{n}$. Then $\Phi_{h}^{1}\left(u_{i}^{v}\right)=\left(M_{i}\right)$ and $k\left(X_{1}, \ldots, X_{n}\right)=k\left(M_{1}, \ldots, M_{n}\right)$. Let $A$ be the discrete valuation ring corresponding to $M_{1}$ in $k\left(M_{1}, \ldots, M_{n}\right)$. One may write the symbols $\left(A_{i}, B_{i}\right)$ as $\left(A_{i}^{\prime}, B_{i}^{\prime}\right)$ where the $A_{i}^{\prime}$ are monomials in $M_{2}, \ldots, M_{n}$ and $B_{i}^{\prime}$ monomials in $M_{1}, \ldots, M_{n}$. Moreover, by taking suitable powers of $B_{i}^{\prime}$ and $A_{i}^{\prime}$, we may assume that $\nu_{A}\left(B_{i}^{\prime}\right) \in\{0,1\}$. Then let $I_{i}^{\prime}$ and $J_{i}^{\prime}$ be generators of $D_{i}$ such that

$$
I_{i}^{\prime p}=A_{i}^{\prime}, J_{i}^{\prime p}=B_{i}^{\prime} \text { and } I_{i}^{\prime} J_{i}^{\prime}=\xi J_{i}^{\prime} I_{i}^{\prime} \text { for } 1 \leqslant i \leqslant m
$$

and let $\mathcal{D}_{i}$ be the order of $D_{i}$ over $A$ generated by $I_{i}^{\prime}$ and $J_{i}^{\prime}$. It is a maximal order of $D_{i}$. Indeed, if $\nu_{A}\left(B_{i}^{\prime}\right)=0$, this follows from the fact that $\mathcal{D}_{i} /\left(M_{1}\right)$ is a skew field. Otherwise, define a function $\nu$ on $D_{i}-\{0\}$ by

$$
\nu\left(\sum_{\substack{u \leqslant j<p \\ 0 \leqslant i<p}} a_{j, l} I_{i}^{j} J_{i}^{l}\right)=\inf _{\substack{0 \leqslant j<p \\ 0 \leqslant l<p}}\left(\nu_{A}\left(a_{j, l}\right)+\frac{l}{p}\right)
$$

then $\nu$ verifies $\nu(a+b) \geqslant \inf (\nu(a), \nu(b))$, and for any $a, b$ in $D_{i}-\{0\}$, write $a=J_{i}^{r} \alpha$ and $b=J_{i}^{s} \beta$ with $\nu(\alpha)=\nu(\beta)=0$. Then

$$
\alpha=\sum_{\substack{0 \leqslant j<p \\ 0 \leqslant l<p}} \alpha_{j, l} I_{i}^{j} J_{i}^{l} \text { and } \beta=\sum_{\substack{0 \leqslant j<p \\ 0 \leqslant i<p}} \beta_{j, l} I_{i}^{j} J_{i}^{l}
$$

with $\inf _{0 \leqslant j<p} \nu_{A}\left(\alpha_{j, 0}\right)=\inf _{0 \leqslant j<p} \nu_{A}\left(\beta_{j, 0}\right)=0$. Since $A\left[A_{i}^{\prime 1 / p}\right] /\left(\mathfrak{m}_{A}\right)$ is a field, we get that $\nu\left(\sum_{0 \leqslant j, l<p}\left(\alpha_{j, 0} \beta_{l, 0}\right) I_{i}^{j+l}\right)=0$ and then that $\nu(a b)=\nu(a)+\nu(b)$. Thus $\nu$ is a valuation and $\mathcal{D}_{i}$ the maximal order of $D_{i}$. Let $\mathcal{Y}_{i}$ be the connected component of the corresponding Severi-Brauer scheme on Spec $A$ which contains the generic fiber (see [Art], [Brz] and [Fr, page 37]). This is Artin's model of $Y_{i}$. Let $\mathcal{Y}$ be the products of the $\mathcal{Y}_{i}$. If $M_{1}$ does not divide $A_{i}^{\prime}$ then the special fiber $\mathcal{Y}_{i}^{u}$ of $\mathcal{Y}_{i}$ is the Severi-Brauer variety corresponding to the algebra $\mathcal{D}_{i} \otimes \kappa_{A}$ and otherwise, by [Art, theorem 1.4], $\mathcal{Y}_{i}^{0^{s}}$ has $p$ irreducible components birationally equivalent to $\mathbf{P}_{\kappa^{s}}^{p-1}$. They may be described as follows: $\mathcal{D}_{i} \otimes \kappa_{A}$ is the algebra generated by $I_{i}^{\prime}$ and $J_{i}^{\prime}$ with the relations

$$
I_{i}^{\prime p}=A_{i}^{\prime}, J_{i}^{\prime p}=0 \text { and } I_{i}^{\prime} J_{i}^{\prime}=\xi J_{i}^{\prime} I_{i}^{\prime}
$$


Let us consider the algebra $\mathcal{D}_{i}^{\prime}=\mathcal{D}_{i} \otimes \kappa_{A}\left(A_{i}^{\prime 1 / p}\right)$. A maximal set of orthogonal idempotents $e_{j}$ of $\mathcal{D}_{i}^{\prime}$ is given by

$$
e_{j}=\prod_{\substack{i \in Z / p \mathrm{Z} \\ l \neq j}} \frac{I_{i}^{\prime}-\xi^{l} A_{i}^{\prime 1 / p}}{\left(\xi^{j}-\xi^{l}\right) A_{i}^{\prime 1 / p}} .
$$

This follows from the existence of an isomorphism from $D_{i} \times K\left(A_{i}^{\prime 1 / p}\right)$ onto $M_{p}\left(K^{-}\left(A_{i}^{\prime 1 / p}\right)\right)$ which maps $I_{i}$ to the diagonal matrice $D\left(A_{i}^{\prime 1 / p}, \xi A_{i}^{\prime 1 / p}, \ldots, \xi^{p-1} A_{i}^{\prime 1 / p}\right)$. Therefore the points of $\mathcal{Y}_{i}^{0^{s}}$ which correspond to right ideals $L$ for which $\operatorname{dim} L e_{j}=1$ (see [Art, lemma 3.3]) are given by $d e_{j} \mathcal{D}_{i}^{\prime}$ for $d$ in $\mathcal{D}_{i}^{\prime}$ such that $d e_{j}$ belongs to $\mathcal{D}_{i}^{\prime}-\{0\}$ and $1 \leq j \leq p$. Therefore the components of $\mathcal{Y}_{i}^{0^{s}}$ are permuted cyclically by $\mathrm{Gal}\left(\kappa_{A}\left(A_{i}^{1 / p}\right) / \kappa_{A}\right)$. Thus the special fiber $\mathcal{Y}^{0}$ of $\mathcal{Y}$ is integral over $\kappa_{A}$ and defines a discrete valuation ring $B$ over $A$ which is unramified over $A$.

We may reduce to the case where $M_{1} \mid A_{i}^{\prime}$ if and only if $1 \leqslant i \leqslant l$ for some $l$ between 1 and $m$. Then $\kappa_{B}$ is rational over

$$
\kappa_{A}\left(\left(B_{i}^{\prime}\right)^{1 / p}, 1 \leqslant i \leqslant l\right)\left(\prod_{l+1 \leqslant i \leqslant m} \mathcal{Y}_{i}^{0}\right) .
$$

By $[\mathrm{Pe} 1$, lemma 6$]$

$$
\partial_{B}\left(\Phi_{k(V), X}^{3}(\lambda)\right)=\Phi_{\kappa_{B}}^{2}\left(\hat{u}_{1}^{\prime}(\lambda)\right)
$$

where $\Phi_{\kappa_{B}}^{*}: \Lambda^{*} u_{1}^{\prime}{ }^{\perp} \rightarrow H^{*}\left(\kappa_{B}, \mu_{p}^{\otimes *}\right)$ is the morphism which sends $u_{i}^{\prime}$ over $M_{i}$ for $2 \leqslant i \leqslant n$ and for any $w$ of $\Lambda^{i} U^{\vee}, \hat{u}_{1}^{\prime}(w)$ is the unique element of $\Lambda^{i-1}\left(u_{1}^{\prime}{ }^{\perp}\right)$ such that

$$
w-u_{1}^{\prime \vee} \wedge \hat{u}_{1}^{\prime}(w) \in \Lambda^{i}\left(u_{1}^{\prime \perp}\right)
$$

We want to show that the image of $\lambda$ is ramified at $B$. Therefore, we shall now describe the kernel of $\Phi_{\kappa_{B}}^{2}$.

Lemma 9.5. With notation as above,

$$
\operatorname{Ker}\left(\Phi_{\kappa_{B}}^{2}\right)=\hat{u}_{1}^{\prime}\left(V^{\vee}\right) \wedge\left(u_{1}^{\prime \perp}\right)+V^{\vee} \cap \Lambda^{2}\left(u_{1}^{\prime \perp}\right) .
$$

Proof. Let $a_{i}$ (resp. $b_{i}$ ) be the inverse image of $\left(A_{i}^{\prime}\right)$ (resp. $\left.\left(B_{i}^{\prime}\right)\right)$ in $U^{\vee}$. Then the right hand side coincides with

$$
\sum_{i=1}^{l} b_{i} \wedge\left(u_{1}^{\prime \perp}\right)+\sum_{i=l+1}^{m} \mathbf{F}_{p} a_{i} \wedge b_{i}
$$

which is contained in $\operatorname{Ker} \Phi_{\kappa_{B}}^{2}$. Moreover Amitsur's theorem implies (see [Pe1, lemma 8]) that

$$
\operatorname{Ker}\left(H^{2}\left(\kappa_{A}\left(\left(B_{i}^{\prime}\right)^{1 / p}, 1 \leqslant i \leqslant l\right), \mu_{p}^{\otimes 2}\right) \rightarrow H^{2}\left(\kappa_{B}, \mu_{p}^{\otimes 2}\right)\right)
$$

is generated by the symbols $\left(A_{i}^{\prime}, B_{i}^{\prime}\right)$ for $i \geqslant l+1$. Therefore we only have to consider the case $m=l$.

In this case, let $w_{1}^{\vee}, \ldots w_{r}^{\vee}$ be a basis of $\tilde{u}_{1}^{\prime}\left(V^{\vee}\right)$. We complete it in a basis $\left(w_{i}^{\vee}\right)_{1 \leqslant i \leqslant m-1}$ of $u_{1}^{\prime}{ }^{\perp}$. Let $w_{1}, \ldots, w_{m-1}$ be the dual basis and let $\eta$ belong to

$$
\Lambda^{2}\left(u_{1}^{\prime \perp}\right)-\hat{u}_{1}^{\prime}\left(V^{\vee}\right) \wedge\left(u_{1}^{\prime \perp}\right) \text {. }
$$

Then there exist $i$ and $j$ strictly bigger than $r$ such that

$$
<\eta \mid w_{i} \wedge w_{j}>\neq 0
$$


We may construct a discrete valuation ring $A^{\prime}\left(\right.$ resp. $\left.B^{\prime}\right)$ in $k\left(M_{2}, \ldots, M_{n}\right)$ (resp. $\left.\kappa_{B}\right)$ such that

$$
\nu_{A^{\prime}}\left(\Phi_{\kappa_{A}}^{1}\left(w_{k}\right)\right)=\delta_{i, k} \text { and } \kappa_{A^{\prime}}=k\left(N_{1}, \ldots, \hat{N}_{i}, \ldots, N_{n-1}\right)
$$

where $N_{1}, \ldots, N_{n-1}$ are monomials corresponding to $w_{1}, \ldots, w_{n-1}$, such that $B^{\prime}$ is unramified on $A^{\prime}$, which is possible since $i>r$ and

$$
\kappa_{B^{\prime}}=k\left(N_{1}, \ldots, N_{i}, \ldots, N_{n}\right)\left(N_{1}^{1 / p}, \ldots, N_{r}^{1 / p}\right) .
$$

Thus

$$
\partial_{B^{\prime}}\left(\Phi_{\kappa_{B}}^{2}(\eta)\right)=\Phi_{\kappa_{B^{\prime}}}^{1}\left(\sum_{j \neq i}<\eta \mid w_{i} \wedge w_{j}>w_{j}\right)=\left(\prod_{j \neq i} N_{j}^{\left\langle\eta \mid w_{i} \wedge w_{j}\right\rangle}\right) \neq 0
$$

and

$$
\Phi_{\kappa_{B}}^{2}(\eta) \neq 0
$$

End of the proof of proposition 9.4. We want to show that $\Phi_{\kappa_{B}}^{2}\left(\hat{u}_{1}^{\prime}(\lambda)\right)$ is non zero, which, by lemma 9.5 , is equivalent to

$$
\hat{u}_{1}^{\prime}(\lambda) \notin \hat{u}_{1}^{\prime}\left(V^{\vee}\right) \wedge u_{1}^{\prime \perp}+V^{\vee} \cap \Lambda^{2}\left(u_{1}^{\perp}\right) .
$$

Taking the inverse image by $\hat{u}_{1}^{\prime}$ is is sufficient to show that

$$
\lambda \notin V^{\vee} \wedge u_{1}^{\prime \perp}+V^{\vee} \wedge u_{1}^{\prime \vee}+\Lambda^{3}\left(u_{1}^{\prime \perp}\right)=V^{\vee} \wedge U^{\vee}+\Lambda^{3}\left(u_{1}^{\prime \perp}\right) .
$$

But, by hypothesis, $p \wedge u_{1}^{\prime}$ belongs to $\left(V^{\vee} \wedge U^{\vee}\right)^{\perp}$. Thus $p \wedge u_{1}^{\prime}$ is zero on

$$
V^{\vee} \wedge U^{\vee}+\Lambda^{3}\left(u_{1}^{\prime \perp}\right)
$$

Since $<\lambda, p \wedge u_{1}^{\prime}>\neq 0$, the result is proved.

9.3. An explicit example. We now assume that $U$ is an $\mathbf{F}_{2}$ vector space of dimension 6 and $V$ is the dual of the subspace of $\Lambda^{2} U$ generated by the elements

$$
u_{2}^{\vee} \wedge u_{5}^{\vee}, u_{4}^{\vee} \wedge u_{1}^{\vee}, u_{6}^{\vee} \wedge u_{3}^{\vee},\left(u_{2}^{\vee}+u_{4}^{\vee}+u_{6}^{\vee}\right) \wedge\left(u_{1}^{\vee}+u_{3}^{\vee}+u_{5}^{\vee}\right) .
$$

Lemma 9.6. With the notations of section 9.2

$$
K_{\max }^{\mathbf{3}} / V^{\vee} \wedge U^{\vee} \sim \mathbf{Z} / 2 \mathbf{Z}
$$

Proof. We have

$$
\begin{aligned}
V^{\vee} \wedge U^{\vee}= & <u_{1}^{\vee} \wedge u_{2}^{\vee} \wedge u_{4}^{\vee}, u_{1}^{\vee} \wedge u_{2}^{\vee} \wedge u_{5}^{\vee}, u_{1}^{\vee} \wedge u_{3}^{\vee} \wedge u_{4}^{\vee}, \\
& u_{1}^{\vee} \wedge u_{3}^{\vee} \wedge u_{6}^{\vee}, u_{1}^{\vee} \wedge u_{4}^{\vee} \wedge u_{5}^{\vee}, u_{1}^{\vee} \wedge u_{4}^{\vee} \wedge u_{6}^{\vee}, \\
& u_{2}^{\vee} \wedge u_{3}^{\vee} \wedge u_{5}^{\vee}, u_{2}^{\vee} \wedge u_{3}^{\vee} \wedge u_{6}^{\vee}, u_{2}^{\vee} \wedge u_{4}^{\vee} \wedge u_{5}^{\vee}, \\
& u_{2}^{\vee} \wedge u_{5}^{\vee} \wedge u_{6}^{\vee}, u_{3}^{\vee} \wedge u_{4}^{\vee} \wedge u_{6}^{\vee}, u_{3}^{\vee} \wedge u_{5}^{\vee} \wedge u_{6}^{\vee}, \\
& u_{1}^{\vee} \wedge u_{2}^{\vee} \wedge u_{3}^{\vee}+u_{1}^{\vee} \wedge u_{5}^{\vee} \wedge u_{6}^{\vee}, u_{2}^{\vee} \wedge u_{3}^{\vee} \wedge u_{4}^{\vee}+u_{1}^{\vee} \wedge u_{2}^{\vee} \wedge u_{6}^{\vee}, \\
& u_{3}^{\vee} \wedge u_{4}^{\vee} \wedge u_{5}^{\vee}+u_{1}^{\vee} \wedge u_{2}^{\vee} \wedge u_{3}^{\vee}, u_{4}^{\vee} \wedge u_{5}^{\vee} \wedge u_{6}^{\vee}+u_{2}^{\vee} \wedge u_{3}^{\vee} \wedge u_{4}^{\vee}>
\end{aligned}
$$

Thus

$$
\begin{aligned}
\left(V^{\vee} \wedge U^{\vee}\right)^{\perp}=< & u_{1} \wedge u_{2} \wedge u_{3}+u_{3} \wedge u_{4} \wedge u_{5}+u_{5} \wedge u_{6} \wedge u_{1}, \\
& u_{2} \wedge u_{3} \wedge u_{4}+u_{4} \wedge u_{5} \wedge u_{6}+u_{6} \wedge u_{1} \wedge u_{2}, \\
& u_{2} \wedge u_{4} \wedge u_{6}, u_{1} \wedge u_{3} \wedge u_{5}>
\end{aligned}
$$


Let

and

$$
\begin{aligned}
& g_{1}=u_{1} \wedge u_{2} \wedge u_{3}+u_{3} \wedge u_{4} \wedge u_{5}+u_{5} \wedge u_{6} \wedge u_{1}, \\
& g_{2}=u_{2} \wedge u_{3} \wedge u_{4}+u_{4} \wedge u_{5} \wedge u_{6}+u_{6} \wedge u_{1} \wedge u_{2}, \\
& h_{1}=u_{2} \wedge u_{4} \wedge u_{6}
\end{aligned}
$$

$$
h_{2}=u_{1} \wedge u_{3} \wedge u_{5} .
$$

Since

we get that

$$
g_{1}+g_{2}+h_{1}+h_{2}=\left(u_{1}+u_{4}\right) \wedge\left(u_{2}+u_{5}\right) \wedge\left(u_{3}+u_{6}\right),
$$

But by [Pe1, pages 265 and 266]

$$
<h_{1}, h_{2}, g_{1}+g_{2}+h_{1}+h_{2}>\subset\left(V^{\vee} \wedge U^{\vee}\right) \frac{1}{\operatorname{dec}} .
$$

$$
\left(\sum_{i=1}^{6} a_{i} u_{i}\right) \wedge\left(\alpha g_{1}+\beta g_{2}+\gamma h_{1}+\delta h_{2}\right)=0
$$

is equivalent to

$$
\begin{aligned}
& a_{1} \beta+a_{4} \alpha=0 \quad a_{1} \alpha+a_{4} \gamma=0 \\
& a_{2} \gamma+a_{5} \alpha=0 \quad a_{3} \alpha+a_{6} \gamma=0 \\
& a_{3} \beta+a_{6} \alpha=0 a_{3} \delta+a_{6} \beta=0 \\
& a_{1} \delta+a_{4} \beta=0 a_{2} \beta+a_{5} \delta=0 \\
& a_{2} \alpha+a_{5} \beta=0 \text {. }
\end{aligned}
$$

Let us assume that $\alpha=1$ and $\beta=0$. Then $a_{4}=a_{2}=a_{6}=0$ and this implies that $a_{3}=a_{5}=a_{1}=0$. Thus the elements of the form $g_{1}+\gamma h_{1}+\delta h_{2}$ are not decomposable. This is also the case for the elements of the form $g_{2}+\gamma h_{1}+\delta h_{2}$. Thus

$$
\left[g_{1}\right] \in\left(V^{\vee} \wedge U^{\vee}\right)^{\perp} /<h_{1}, h_{2}, g_{1}+g_{2}+h_{1}+h_{2}>
$$

does not lift to a decomposable element. This implies the equality

$$
<h_{1}, h_{2}, g_{1}+g_{2}+h_{1}+h_{2}>=\left(V^{\vee} \wedge U^{\vee}\right)_{\mathrm{dec}}^{\perp} .
$$

Proposition 9.7. With notation as above

$$
\operatorname{Ker}\left(\Phi_{k(V)}^{3}\right)=K_{\max }^{3} \neq V^{\vee} \wedge U^{\vee} .
$$

Proof. The group $K_{\max }^{3}$ is the orthogonal of $\left(V^{\vee} \wedge U^{\vee}\right)_{\text {dec }}^{\perp}$. Therefore we have

$$
K_{\max }^{3}=V^{\vee} \wedge U^{\vee}+<u_{2}^{\vee} \wedge u_{3}^{\vee} \wedge u_{4}^{\vee}+u_{5}^{\vee} \wedge u_{6}^{\vee} \wedge u_{1}^{\vee}>.
$$

Thus it is enough to show that

$$
\left(X_{2}, X_{3}, X_{4}\right)+\left(X_{5}, X_{6}, X_{1}\right)
$$

is trivial in $H^{3}(K(V), \mathbf{Z} / 2 \mathbf{Z})$. Let $V^{\prime}$ be the product of the conics $C_{1}, C_{2}, C_{3}$ given by the homogeneous equations

$$
\begin{aligned}
& \left(C_{1}\right): T_{1,1}^{2}-X_{2} T_{1,2}^{2}-X_{5} T_{1,3}^{2}=0 \\
& \left(C_{2}\right): T_{2,1}^{2}-X_{4} T_{2,2}^{2}-X_{1} T_{2,3}^{2}=0 \\
& \left(C_{3}\right): T_{3,1}^{2}-X_{6} T_{3,2}^{2}-X_{3} T_{3,3}^{2}=0
\end{aligned}
$$

Then a direct computation using the assertion (ii) of proposition 3.4 for quadratic extensions yields an explicit element of $\mathrm{CH}^{2}(V)$ which is either 0 or the unique non trivial 
element of $\mathrm{CH}^{2}(V)_{\text {tors }}$. Then the method described in section 8 used backwards yields the function $f$ of $k\left(V^{\prime}\right)$ defined by

$f=\left(\frac{T_{1,1}}{T_{1,3}}\right)^{2} X_{1}-\left(\frac{T_{2,1}}{T_{2,3}}\right)^{2} X_{5}=\left(\frac{T_{2,1} T_{1,2}}{T_{2,3} T_{1,3}}\right)^{2} X_{2}-\left(\frac{T_{1,1} T_{2,2}}{T_{1,3} T_{2,3}}\right)^{2} X_{4} \in k\left(C_{1} \times \cdots \times C_{3}\right)$.

But by [Lam, chapter 10, proposition 1.3],

$$
\begin{aligned}
\left(f, X_{1} X_{3} X_{5}, X_{2} X_{4} X_{6}\right)= & \left(X_{2}\left(\frac{T_{2,1} T_{1,2}}{T_{2,3} T_{1,3}}\right)^{2}-X_{4}\left(\frac{T_{1,1} T_{2,2}}{T_{1,3} T_{2,3}}\right)^{2}, X_{2} X_{4}, X_{1} X_{3} X_{5}\right) \\
& +\left(X_{1}\left(\frac{T_{1,1}}{T_{1,3}}\right)^{2}-X_{5}\left(\frac{T_{2,1}}{T_{2,3}}\right)^{2}, X_{6}, X_{1} X_{5}\right) \\
= & \left(X_{2}, X_{4}, X_{1} X_{3} X_{5}\right)+\left(X_{1}, X_{5}, X_{6}\right) \\
= & \left(X_{2}, X_{3}, X_{4}\right)+\left(X_{1}, X_{5}, X_{6}\right) .
\end{aligned}
$$

This element is therefore trivial in $H^{3}(k(V), \mathbf{Z} / 2 \mathbf{Z})$.

Corollary 9.8. Consider $U=\bigoplus_{i=1}^{6} \mathbf{F}_{2} u_{i}, V=\bigoplus_{i=1}^{4} \mathbf{F}_{2} v_{i}$ and $G$ the central extension of $U$ by $V$ corresponding to the following element of $H^{2}(U, V)$ :

$$
u_{2}^{\vee} \cup u_{5}^{\vee} \otimes v_{1}+u_{4}^{\vee} \cup u_{1}^{\vee} \otimes v_{2}+u_{6}^{\vee} \cup u_{3}^{\vee} \otimes v_{3}+\left(u_{1}^{\vee}+u_{3}^{\vee}+u_{5}^{\vee}\right) \cup\left(u_{2}^{\vee}+u_{4}^{\vee}+u_{6}^{\vee}\right) \otimes v_{4} .
$$

Then for any field $k$ containing a primitive fourth root of one, $u_{1}^{\vee} \cup u_{2}^{\vee} \cup u_{3}^{\vee}+u_{4}^{\vee} \cup u_{5}^{\vee} \cup u_{6}^{\vee}$ gives a non-trivial $k$-negligible element in $H^{3}(G, \mathbf{Q} / \mathbf{Z})$.

Proof. This follows from proposition 9.7, lemma 9.3 and proposition 9.1.

Corollary 9.9. For any field $k$ of characteristic different from 2 and containing the fourth roots of one, for any family $\left(a_{1}, \ldots, a_{6}\right)$ of elements in $k^{*}$ such that

$$
\left(a_{1}, a_{4}\right)=\left(a_{2}, a_{5}\right)=\left(a_{3}, a_{6}\right)=\left(a_{2} a_{4} a_{6}, a_{1} a_{3} a_{5}\right)=0
$$

one has

$$
\left(a_{1}, a_{2}, a_{3}\right)+\left(a_{4}, a_{5}, a_{6}\right)=0 .
$$

I am very thankful to Markus Rost for several discussions during which I realized that it was possible to generalize the result I had for products of Severi-Brauer varieties to the case of generalized flag varieties.

\section{REFERENCES}

[Am] S. A. Amitsur, Generic splitting fields of central simple algebras, Ann. of Math. 62 (1955), 8-43.

[Ara] J. K. Arason, Cohomologische Invarianten quadratischer Formen, J. Algebra 36 (1975), 448-491.

[Art] M. Artin, Left ideals in maximal orders, Brauer groups in ring theory and algebraic geometry (Antwerp, 1981), Lect. Notes in Math., $\mathrm{n}^{\circ}$ 917, Springer-Verlag, Berlin, Heidelberg and New York, 1982, pp. 182-193.

[Bo] A. Borel, Linear algebraic groups (Second enlarged edition), Graduate Texts in Math., vol. 126, Springer-Verlag, Berlin, Heidelberg and New York, 1991.

[Bki] N. Bourbaki, Groupes et algèbres de Lie. Chap. 4, 5 et 6 , Masson, Paris, 1981

[Bro] K. S. Brown, Cohomology of groups, Graduate Texts in Math., vol. 87, Springer-Verlag, Berlin, Heidelberg and New York, 1982.

[Brz] J. Brzezinski, Brauer-Severi schemes of orders, Orders and their applications (Oberwolfach, 1984) (I. Feiner and K. W. Roggenkamp, eds.), Lecture notes in math., vol. 1142, SpringerVerlag, Berlin, Heidelberg and New York, 1985, pp. 18-49.

[Car1] H. Cartan, Détermination des algèbres $H_{*}\left(\Pi, n ; \mathbf{Z}_{p}\right)$ et $H^{*}\left(\Pi, n ; \mathbf{Z}_{p}\right)$, p premier impair, Séminaire Henri Cartan 1954/55, $\mathbf{n}^{\circ} 9$.

[Car2] - Détermination des algèbres $H_{*}\left(\Pi, n ; \mathbf{Z}_{2}\right)$ et $H^{*}\left(\Pi, n ; \mathbf{Z}_{2}\right)$; groupes stables modulo $p$, Séminaire Henri Cartan 1954/55, n 10. 
[CTO] J.-L. Colliot-Thélène et M. Ojanguren, Variétés unirationnelles non rationnelles: au-dela de l'exemple d'Artin et Mumford, Invent. math. 97 (1989), 141-158.

[CTR] J.-L. Colliot-Thélène and $W$. Raskind, $K_{2}$-cohomology and the second Chow group, Math. Ann. 270 (1985), 165-199.

[De] M. Demazure, Désingularisation des variétés de Schubert généraliséts, Ann. Sci. F́cole Norm. Sup. (4) 7 (1974), 53-88.

[Fr] E. Frossard, Arithmétique des variétés fubrées en variétés de Severi-Brauer au-dessus d'une courbe de genre quelconque, Thèse 1671, Université de Paris-Sud, 1995.

[Fu] W. Fulton, Intersection theory, Ergebnisse der Mathematik und ihrer Grenzgebiete, 3. Folge, vol. 2, Springer-Verlag, Berlin, Heidelberg and New York, 1984.

[HS] G. Hochschild and J.-P. Serre, Cohomology of group extensions, Trans. Amer. Math. Soc. 74 (1953), 110-134.

[Kah] B. Kahn, Descente galoisienne et $K_{2}$ des corps de nombres, K-theory 7 (1993), 55-100.

[Kar] N. A. Karpenko, Algebro-geometric invariants of quadratic forms, Algebra i analiz 2 (1990), $\mathrm{n}^{\circ}$ 1, 141-162; Eriglish transl. in Leningrad Math. J. 2 (1991), $\mathrm{n}^{\circ} 1,119-138$.

[KR] I. Kersten and U. Rehmann, Generic splitting of reductive groups, Tôhoku Math. J. 46 (1994), 35-70.

[Lag] A. Laghribi, Isotropie de certaines formes quadratiques de dimensions 7 et 8 sur le corps des fonctions d'une quadrique, Prépublication, 1995, à paraître au Duke Math. J.

[Lam] T. Y. Lam, The algebraic theory of quadratic forms, Benjamin, Reading, 1973

[Li1] S. Lichtenbaum, Values of zeta-functions at non-negative integers, Number theory (Noordwijkerhout, 1983) (H. Jager, ed.), Lecture Notes in Math., vol. 1068, Springer-Verlag, Berlin, Heidelberg and New York, 1984, pp. 127-138.

[Li2] - The construction of weight-two arithmetic cohomology, Invent. math. 88 (1987), 183 215.

[Li3] - New results on weight-two motivic cohomology, The Grothendieck Festschrift III (P. Cartier, L. Mlusie, N. M. Katz, G. Laumon, Y. Manin, and K. A. Ribet, eds.), Progress in Math., vol. 88, Birkhäuser, Boston, 1990, pp. 35-55.

[Me] A.S. Merkur'ev, The group $H^{1}\left(X, \mathcal{K}_{2}\right)$ for projective homogeneous varieties, Algebra i Analiz 7 (1996); English transl. in St Petersburg Math. J. 7 (1996).

[MPW] A. S. Merkur'ev, I. A. Panin, and A. R. Wadsworth, Index reduction formulas for twisted flag varieties, I, Preprint, 1995.

[MS] A.S. Merkur'ev and A. A. Suslin, $\mathcal{K}$-cohomology of Severi-Brauer varietits and the norm residue homomorphism, Izv. Akad. Nauk SSSR Ser. Mat. 46 (1982), $\mathrm{n}^{\circ}$ 5, 1011-1046; English transl. in Math. USSR-Izv. 21 (1983), $\mathbf{n}^{\circ} 2,307-340$.

[Pa] I. A. Panin, On the algebraic $K$-theory of twisted flag varities, $K^{\circ}$-theory $8(1994), n^{\circ} 6,541-585$.

[Pe1] E. Peyre, Unramified cohomology and rationality problems, Math. Ann. 296 (1993), 247-268.

[Pe2] - Products of Severi-Brauer varieties and Galois cohomology, K-theory and algebraic geometry: connections with quadratic forms and division algebras (Santa-Barbara, 1992) (B. Jacob and A. Rosenberg, eds.), Proc. Sympos. Pure Math., vol. 58.2, AMS, Providence, 1995, pp. 369-401.

[Pe3] - Hauteurs et mesures de Tamagawa sur les variétés de Fano, Duke Math. J. 79 (1995), $\mathrm{n}^{\circ} 1,101-218$.

[Pe4] Corps de fonctions de variétés homogènes et cohomologie galoisienne, C. R. Acad. Sci. Paris Sér. I Math. 321 (1995), 891-896.

[Sh] C. C. Sherman, $\mathcal{K}$-cohomology of regular schemes, Comm. Algebra 7 (1979), $\mathbf{n}^{\circ} 10,999-1027$.

[St] R. Steinberg, On a theorem of Pittie, Topology 14 (1975), 173-177.

[Ti] J. Tits, Représentations linéaires irréductibles d'un groupe réductif sur un corps quelconque, J. Reine Angew. Math. 247 (1971), 196-220. 



\section{How to connect to the preprint-server of the Institut de Recherche Mathématique Avancée Strasbourg}

The best way is to use Mosaic or any WWW client-program (like Netscape, Macweb, XMosaic, etc.). The URL of this site is

$$
\text { http://galois.u-strasbg.fr/(french home page) }
$$

or

http://galois.u-strasbg.fr/home.html (english home page).

Another way is to use a ftp command in a UNIX-like environment (Telnet on Macintosh, any command-tool on X-Windows, etc.). The command

$$
\text { ftp galois.u-strasbg.fr }
$$

will connect you to the server (login : anonymous, password : your e-mail address). The preprints of the year $19 \mathrm{XX}$ are located in the directory

$$
/ \text { preprint/sxbXX }
$$

Once you found the file you want, you can get a copy by entering first

$$
\text { binary }
$$

The command

$$
\text { get filename }
$$

will transfer the file to your current local directory. You can leave $\mathrm{ftp}$ with

$$
\text { bye }
$$

when you are finished. 
